\title{
Modelling the local and global cloud formation on HD 189733b
}

\author{
E. Lee ${ }^{1}$, Ch. Helling ${ }^{1}$, I. Dobbs-Dixon ${ }^{2}$, and D. Juncher ${ }^{3}$ \\ ${ }^{1}$ SUPA, School of Physics and Astronomy, University of St Andrews, North Haugh, St Andrews, Fife KY16 9SS, UK \\ 2 NYU Abu Dhabi, PO Box 129188, Abu Dhabi, UAE \\ ${ }^{3}$ Niels Bohr Institute \& Centre for Star and Planet Formation, University of Copenhagen, 2100 Copenhagen, Denmark \\ Received 27 February 2015 / Accepted 21 May 2015
}

\begin{abstract}
Context. Observations suggest that exoplanets such as HD 189733b form clouds in their atmospheres which have a strong feedback onto their thermodynamical and chemical structure, and overall appearance.

Aims. Inspired by mineral cloud modelling efforts for brown dwarf atmospheres, we present the first spatially varying kinetic cloud model structures for HD 189733b.

Methods. We apply a 2-model approach using results from a 3D global radiation-hydrodynamic simulation of the atmosphere as input for a detailed, kinetic cloud formation model. Sampling the 3D global atmosphere structure with 1D trajectories allows us to model the spatially varying cloud structure on HD 189733b. The resulting cloud properties enable the calculation of the scattering and absorption properties of the clouds.

Results. We present local and global cloud structure and property maps for HD 189733b. The calculated cloud properties show variations in composition, size and number density of cloud particles which are strongest between the dayside and nightside. Cloud particles are mainly composed of a mix of materials with silicates being the main component. Cloud properties, and hence the local gas composition, change dramatically where temperature inversions occur locally. The cloud opacity is dominated by absorption in the upper atmosphere and scattering at higher pressures in the model. The calculated $8 \mu \mathrm{m}$ single scattering albedo of the cloud particles are consistent with Spitzer bright regions. The cloud particles scattering properties suggest that they would sparkle/reflect a midnight blue colour at optical wavelengths.
\end{abstract}

Key words. planets and satellites: individual: HD 189733b - planets and satellites: atmospheres - methods: numerical

\section{Introduction}

The atmospheres of exoplanets are beginning to be characterised in great detail. Spectroscopic measurements from primary and secondary transits of hot Jupiters have opened up the first examinations into the composition of their atmospheres. HD 189733b is one of the most studied hot Jupiters due to its bright parent star and large planet-to-star radius ratio. Observational data of this object has been collected by various groups from X-ray to radio wavelengths (Knutson et al. 2007; Tinetti et al. 2007; Charbonneau et al. 2008; Grillmair et al. 2008; Swain et al. 2008, 2009; Danielski et al. 2014). Transit spectra from the Hubble Space Telescope (Lecavelier Des Etangs et al. 2008; Sing et al. 2011; Gibson et al. 2012) between $0.3 \mu \mathrm{m}$ and $1.6 \mu \mathrm{m}$ show a featureless spectrum consistent with Rayleigh scattering from the atmosphere. These observations sample the outer limbs (dayside-nightside terminator regions) of the planet's atmosphere during primary eclipse. Lecavelier Des Etangs et al. (2008) suggests haze/clouds to be responsible for the Rayleigh slope and estimate cloud particle radii of $10^{-2} \ldots 10^{-1} \mu \mathrm{m}$ at $\sim 10^{-6} \ldots 10^{-3}$ bar local gas pressures. They suggest $\mathrm{MgSiO}_{3}[\mathrm{~s}]$ as the most likely cloud particle material due to its strong scattering properties. Pont et al. (2013) who reanalysed and combined the Hubble and Spitzer (Agol et al. 2010; Désert et al. 2011; Knutson et al. 2012) observations found that the spectrum was "Dominated by Rayleigh scattering over the whole visible and near-infrared range..." and also suggest cloud/haze layers as a likely scenario. Subsequent analysis by Wakeford \& Sing (2015) showed that single composition cloud condensates could fit the Rayleigh scattering slope with grain sizes of $0.025 \mu \mathrm{m}$. Evans et al. (2013) measured the geometric albedo of HD 189733b over $0.29 \ldots 0.57 \mu \mathrm{m}$ and infer the planet is likely to be a deep blue colour at visible wavelengths, which they attribute to scattering mid-altitude clouds. These albedo observations sample the dayside face of the planet by comparing measurements before, during and after the secondary transit (occulation). Star spots (McCullough et al. 2014), low atmospheric metallicity (Huitson et al. 2012) and photo-chemical, upper atmosphere haze layers (Pont et al. 2013) have been proposed as alternative explanations to these observations.

Clouds present in optically thin atmospheric regions are thought to significantly impact the observed spectra of exoplanets by flattening the ultra-violet and visible spectrum through scattering by small cloud particles, by depletion of elements and by providing an additional opacity source (e.g. GJ 1214b; Kreidberg et al. 2014). The strong radiative cooling (or heating) resulting from the high opacity of the cloud particles also affect the local pressure which influences the local velocity field (Helling et al. 2004). These effects have been observed and modelled in brown dwarf atmospheres which are the inspiration for the current study. Clouds are also important for ionisation of the atmosphere by dust-dust collisions (Helling et al. 2011) and cosmic ray ionisation (Rimmer \& Helling 2013). This leaves open the possibility of lightning discharge events in 
exo-clouds (Bailey et al. 2014). Furthermore, Stark et al. (2014) suggest that charged dust grains could aid the synthesis of prebiotic molecules by enabling the required energies of formation on their surfaces. Multi-dimensional atmosphere simulations have been used to gain a first insight into the atmospheric dynamics of HD 189733 b (e.g. Showman et al. 2009; Fortney et al. 2010; Knutson et al. 2012; Dobbs-Dixon \& Agol 2013). Output from Showman et al. (2009) has been used for kinetic, non-equilibrium photo-chemical models of HD 189733b (Moses et al. 2011; Venot et al. 2012; Agúndez et al. 2014).

In this work, we present cloud structure calculations for HD 189733b based on 3D radiation-hydrodynamic (3D RHD) atmosphere results of Dobbs-Dixon \& Agol (2013). With this 2-model approach, we investigate how the specific cloud properties vary locally and globally throughout HD 189733b's atmosphere. In Sect. 2 we outline our 2-model approach. We summarise our cloud formation model and discuss our approach to atmospheric mixing, cloud particle opacities and model limitations. In Sect. 3 the spatially varying cloud properties (particle sizes, number density, material composition, nucleation rate, growth rate and opacity) are presented. In Sect. 4 we use the results of the locally and spatially varying cloud properties to calculate the wavelength dependent opacity and contribution of the clouds to the reflected light and transit spectra for HD 189733b. Section 5 discusses our results with respect to observational findings for HD 189733b. Our conclusions are outlined in Sect. 6.

\section{2-model approach}

We apply our kinetic dust cloud formation model to results from 3D RHD simulations of HD 189733b's atmosphere (DobbsDixon \& Agol 2013) and present a first study of spatially varying cloud formation on a hot Jupiter. We briefly summarise the main features of the kinetic cloud formation model (see: Woitke \& Helling 2003, 2004; Helling et al. 2008b; Helling \& Fomins 2013). This cloud model has been successfully combined with 1D atmosphere models (DRIFT-PHOENIX; Dehn et al. 2007; Helling et al. 2008a; Witte et al. 2009, 2011) to produce synthetic spectra of M dwarfs, brown dwarfs and non-irradiated hot Jupiter exoplanets. We then summarise the multi-dimensional radiative-hydrodynamical model from Dobbs-Dixon \& Agol (2013) used as input for the kinetic cloud formation model. Finally, we outline our approach in calculating the absorption and scattering properties of multi-material, multi-disperse, mixed cloud particles.

\subsection{Cloud formation modelling}

Cloud formation proceeds via a sequence of processes that are described kinetically in our cloud formation model:

1. Formation of seed particles by homomolecular homogeneous nucleation (Jeong et al. 2003; Lee et al. 2015).

2. Growth of various solid materials by gas-grain chemical surface reactions on the existing seeds or grains (Gail \& Sedlmayr 1986; Helling \& Woitke 2006; Helling et al. 2008b).

3. Evaporation of grains when the materials that they are composed of become thermally unstable (Helling \& Woitke 2006; Helling et al. 2008b).

4. Gravitational settling allowing a continuation of grain growth through transport of grains out of under-saturated regions (Woitke \& Helling 2003, 2004).
5. Element depletion in regions of cloud formation which can stop new grains from forming (Helling \& Woitke 2006).

6. Convective/turbulent mixing from deeper to higher atmospheric regions to provide element replenishment (Woitke \& Helling 2003, 2004).

Without the nucleation step 1, grains do not form and a stationary atmosphere remains dust-free. We refer the reader to Helling \& Fomins (2013) for a summary of the applied theoretical approach to model cloud formation in an oxygen-rich gas. The nucleation rate (formation of seed particles) $J_{*}\left[\mathrm{~cm}^{-3} \mathrm{~s}^{-1}\right]$ for homomolecular homogeneous nucleation (Helling \& Woitke 2006) is

$$
\begin{aligned}
J_{*}(t, \boldsymbol{r})= & \frac{f(1, t)}{\tau_{\mathrm{gr}}\left(1, N_{*}, t\right)} Z\left(N_{*}\right) \\
& \times \exp \left(\left(N_{*}-1\right) \ln S(T)-\frac{\Delta G\left(N_{*}\right)}{R T}\right),
\end{aligned}
$$

where $f(1, t)$ is the number density of the seed forming gas species, $\tau_{\text {gr }}$ the growth timescale of the critical cluster size $N_{*}$, $Z\left(N_{*}\right)$ the Zeldovich factor, $S(T)$ the supersaturation ratio and $\Delta G\left(N_{*}\right)$ the Gibbs energy of the critical cluster size. We consider the homogeneous nucleation of $\mathrm{TiO}_{2}$ seed particles. A new value for the surface tension $\sigma_{\infty}=480\left[\mathrm{erg} \mathrm{cm}^{-2}\right]$ is used based on updated $\mathrm{TiO}_{2}$ cluster calculations from Lee et al. (2015).

The net growth/evaporation velocity $\chi^{\text {net }}\left[\mathrm{cm} \mathrm{s}^{-1}\right]$ of a grain (after nucleation) due to chemical surface reactions (Helling \& Woitke 2006) is

$$
\chi^{\mathrm{net}}(\boldsymbol{r})=\sqrt[3]{36 \pi} \sum_{s} \sum_{r=1}^{R} \frac{\Delta V_{r} n_{r} v_{r}^{\mathrm{rel}} \alpha_{r}}{v_{r}^{\mathrm{key}}}\left(1-\frac{1}{S_{r}} \frac{1}{b_{\text {surf }}^{s}}\right),
$$

where " $r$ " is the index for the chemical surface reaction, $\Delta V_{r}$ the volume increment of the solid " $s$ " by reaction $r, n_{r}$ the particle density of the reactant in the gas phase, $v_{r}^{\text {rel }}$ the relative thermal velocity of the gas species taking part in reaction $r, \alpha_{r}$ the sticking coefficient of reaction $r$ and $v_{r}^{\text {key }}$ the stoichiometric factor of the key reactant in reaction $r$ (Helling \& Woitke 2006). $S_{r}$ is the reaction supersaturation ratio and $1 / b_{\text {surf }}^{s}=V_{s} / V_{\text {tot }}$ the volume ratio of solid s. Our cloud formation method allows the formation of mixed heterogeneous grain mantles. We simultaneously consider 12 solid growth species $\left(\mathrm{TiO}_{2}[\mathrm{~s}], \mathrm{Al}_{2} \mathrm{O}_{3}[\mathrm{~s}]\right.$, $\mathrm{CaTiO}_{3}[\mathrm{~s}], \mathrm{Fe}_{2} \mathrm{O}_{3}[\mathrm{~s}], \mathrm{FeS}[\mathrm{s}], \mathrm{FeO}[\mathrm{s}], \mathrm{Fe}[\mathrm{s}], \mathrm{SiO}[\mathrm{s}], \mathrm{SiO}_{2}[\mathrm{~s}]$, $\mathrm{MgO}[\mathrm{s}], \mathrm{MgSiO}_{3}[\mathrm{~s}], \mathrm{Mg}_{2} \mathrm{SiO}_{4}[\mathrm{~s}]$ ) with 60 surface chemical reactions (Helling et al. 2008b). The local dust number density $n_{\mathrm{d}}\left[\mathrm{cm}^{-3}\right]$ and mean grain radius $\langle a\rangle[\mathrm{cm}]$ are given by

$$
\begin{aligned}
n_{\mathrm{d}}(\boldsymbol{r}) & =\rho_{\mathrm{gas}}(\boldsymbol{r}) L_{0}(\boldsymbol{r}), \\
\langle a\rangle(\boldsymbol{r}) & =\sqrt[3]{\frac{3}{4 \pi}} \frac{L_{1}(\boldsymbol{r})}{L_{0}(\boldsymbol{r})},
\end{aligned}
$$

respectively; where $\rho_{\text {gas }}(\boldsymbol{r})\left[\mathrm{g} \mathrm{cm}^{-3}\right]$ is the local gas volume density and $L_{0}(\boldsymbol{r}), L_{1}(\boldsymbol{r})$ are derived by solving for $L_{j}(\boldsymbol{r})\left[\mathrm{cm}^{j} \mathrm{~g}^{-1}\right]$ in the dust moment equations (Woitke \& Helling 2003).

\section{2. $3 D$ radiative-hydrodynamical model}

The 3D radiative-hydrodynamical [3D RHD] model solves the fully compressible Navier-Stokes equations coupled to a wavelength dependent two-stream radiative transfer scheme. The model assumes a tidally-locked planet with $\phi=0^{\circ}, \theta=0^{\circ}$ denoting the sub-stellar point (the closest point to the host star). There 

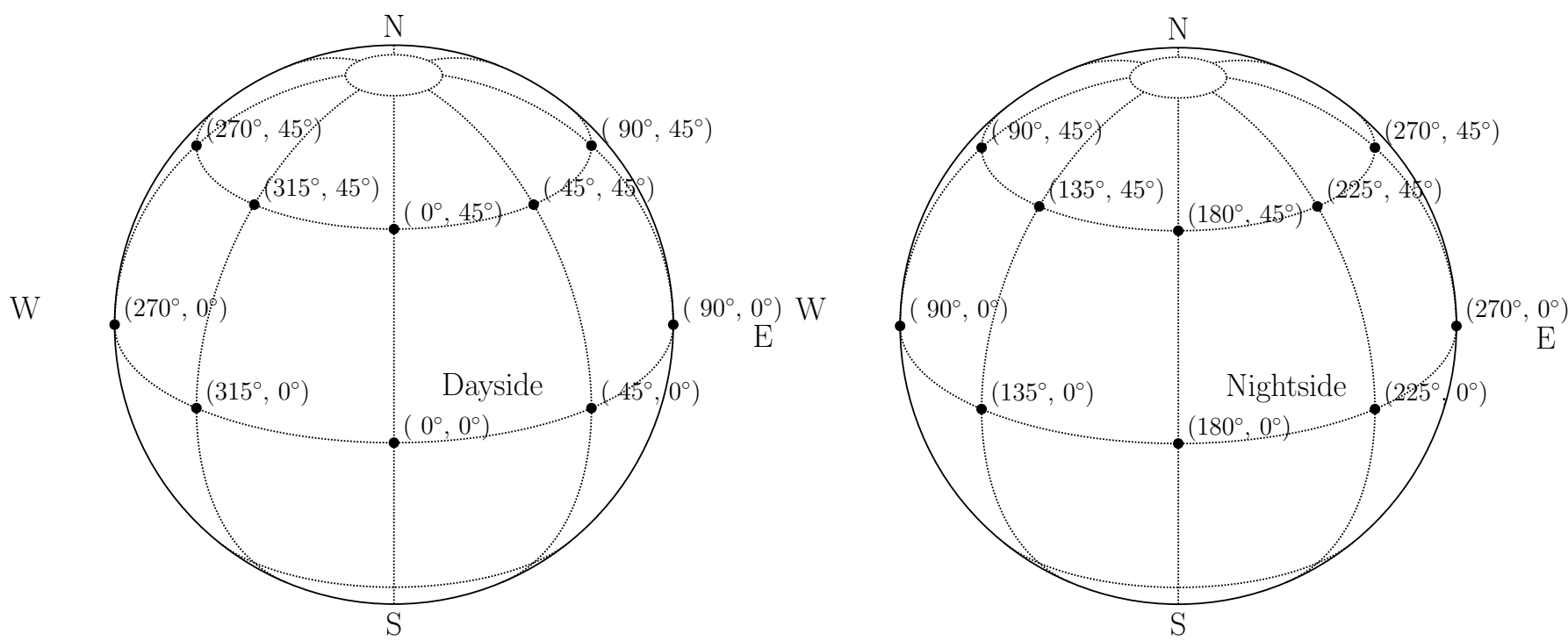

Fig. 1. Illustration of the sample trajectories (black points) taken from the 3D radiative-hydrodynamic model atmosphere of HD 189733b (DobbsDixon \& Agol 2013); longitudes $\phi=0^{\circ} \ldots 315^{\circ}$ in steps of $\Delta \phi=+45^{\circ}$, latitudes $\theta=0^{\circ},+45^{\circ}$. The sub-stellar point is at $\phi=0^{\circ}, \theta=0^{\circ}$. We assume that the sample trajectories are latitudinal (north-south) symmetric.

are three components to the wavelength dependent opacities: molecular opacities consistent with solar composition gas, a gray component representing a strongly absorbing cloud deck, and a strong Rayleigh scattering component. Equations are solved on a spherical grid with pressures ranging from $\sim 10^{-4.5}$ to $\sim 10^{3}$ bar. Input parameters were chosen to represent the bulk observed properties of HD 189733b, but (with the exception of the opacity) were not tuned to match spectroscopic observations. The dominate dynamical feature is the formation of a super-rotating, circumplanetary jet (Tsai et al. 2014) that efficiently advects energy from day to night near the equatorial regions. This jet forms from the planetary rotation (Rossby waves) coupled with eddies which pump positive angular momentum towards the equator (Showman \& Polvani 2011). A counter-rotating jet is present at higher latitudes. Significant vertical mixing, discussed later, is seen throughout the atmosphere. Calculated transit spectra, emission spectra, and light curves agree quite well with current observations from $0.3 \mu \mathrm{m}$ to $8.0 \mu \mathrm{m}$. Further details can be found in Dobbs-Dixon \& Agol (2013).

\subsection{Model set-up and input quantities}

We sampled vertical trajectories of the 3D RHD model at longitudes of $\phi=0^{\circ} \ldots 315^{\circ}$ in steps of $\Delta \phi=+45^{\circ}$ and latitudes of $\theta=0^{\circ},+45^{\circ}$ (Fig. 1). The horizontal wind velocity moves in the positive $\phi$ direction. Figure 2 shows the $\left(T_{\text {gas }}, p_{\text {gas }}\right)$ and vertical velocity profiles used to derive the cloud structure at the equator $\theta=0^{\circ}$ and latitude $\theta=45^{\circ}$. Local temperature inversions are present in the dayside $\left(T_{\text {gas }}, p_{\text {gas }}\right)$ profiles which are exposed to the irradiation of the host star without the need for an additional opacity source. The local temperature maximum migrates eastward with increasing atmospheric depth. This is due to hydrodynamical flows funnelling gas towards the equator causing viscous, compressive and shock heating. The $\left(T_{\text {gas }}, p_{\text {gas }}\right)$ profile at latitude $\theta=45^{\circ}$ has steeper temperature inversions on the dayside. Sample trajectories converge to equal temperatures at $p_{\text {gas }}>1$ bar for all longitudes and latitudes. We apply a 3-point boxcar smoothing to the vertical velocity profiles in order to reduce the effects of unresolved turbulence. Further required input quantities are a constant surface gravity of $\log g=3.32$ and
Table 1. Input quantities for the cloud formation model. Local $T_{\text {gas }}, p_{\text {gas }}$, $\rho_{\text {gas }}, v_{z}$ and $z$ are taken from the $3 \mathrm{D}$ radiation-hydrodynamic model.

\begin{tabular}{lll}
\hline \hline Input & Definition & Units \\
\hline$T_{\text {gas }}(\boldsymbol{r})$ & local gas temperature & $\mathrm{K}$ \\
$p_{\text {gas }}(\boldsymbol{r})$ & local gas pressure & $\mathrm{dyn} \mathrm{cm}-2$ \\
$\rho_{\text {gas }}(\boldsymbol{r})$ & local gas density & $\mathrm{g} \mathrm{cm}^{-3}$ \\
$v_{z}(\boldsymbol{r})$ & local vertical gas velocity & $\mathrm{cm} \mathrm{s}^{-1}$ \\
$z$ & vertical atmospheric height & $\mathrm{cm}$ \\
$\epsilon_{\mathrm{x}}^{0} / \epsilon_{\mathrm{H}(\boldsymbol{r})}$ & element abundance & - \\
$g(\boldsymbol{r})$ & surface gravity & $\mathrm{cm} \mathrm{s}^{-2}$ \\
\hline
\end{tabular}

initial solar element abundances $\epsilon_{\mathrm{x}}^{0} / \epsilon_{\mathrm{H}}$ (element "x" to Hydrogen ratio) with $\mathrm{C} / \mathrm{O}=0.427$ (Anders \& Grevesse 1989) for all atmospheric layers. However, we note the element abundance of the gas phase will increase or decrease due to cloud formation or evaporation (Fig. 8). We assume local thermal equilibrium (LTE) for all gas-gas and dust-gas chemical reactions. The required input quantities for the kinetic cloud formation model are summarised in Table 1.

\subsection{Atmospheric vertical mixing}

Vertical mixing is important to resupply the upper atmosphere with elements which have been depleted by cloud particle formation and their subsequent gravitational settling into deeper atmospheric layers (Woitke \& Helling 2003). Without mixing, the cloud particles in the atmosphere would rain out and remove heavy elements from the upper atmosphere. This leaves a metal poor gas phase where no future cloud particles could form (Woitke \& Helling 2004; Appendix A).

\subsubsection{Previous brown dwarf approach}

The main energy transport in the core of a brown dwarf is convection. The atmosphere is convectively unstable in the inner, hotter parts. This atmospheric convection causes substantial overshooting into even higher, and radiation dominated parts (e.g. Ludwig et al. 2002). Woitke \& Helling $(2003,2004)$ define 

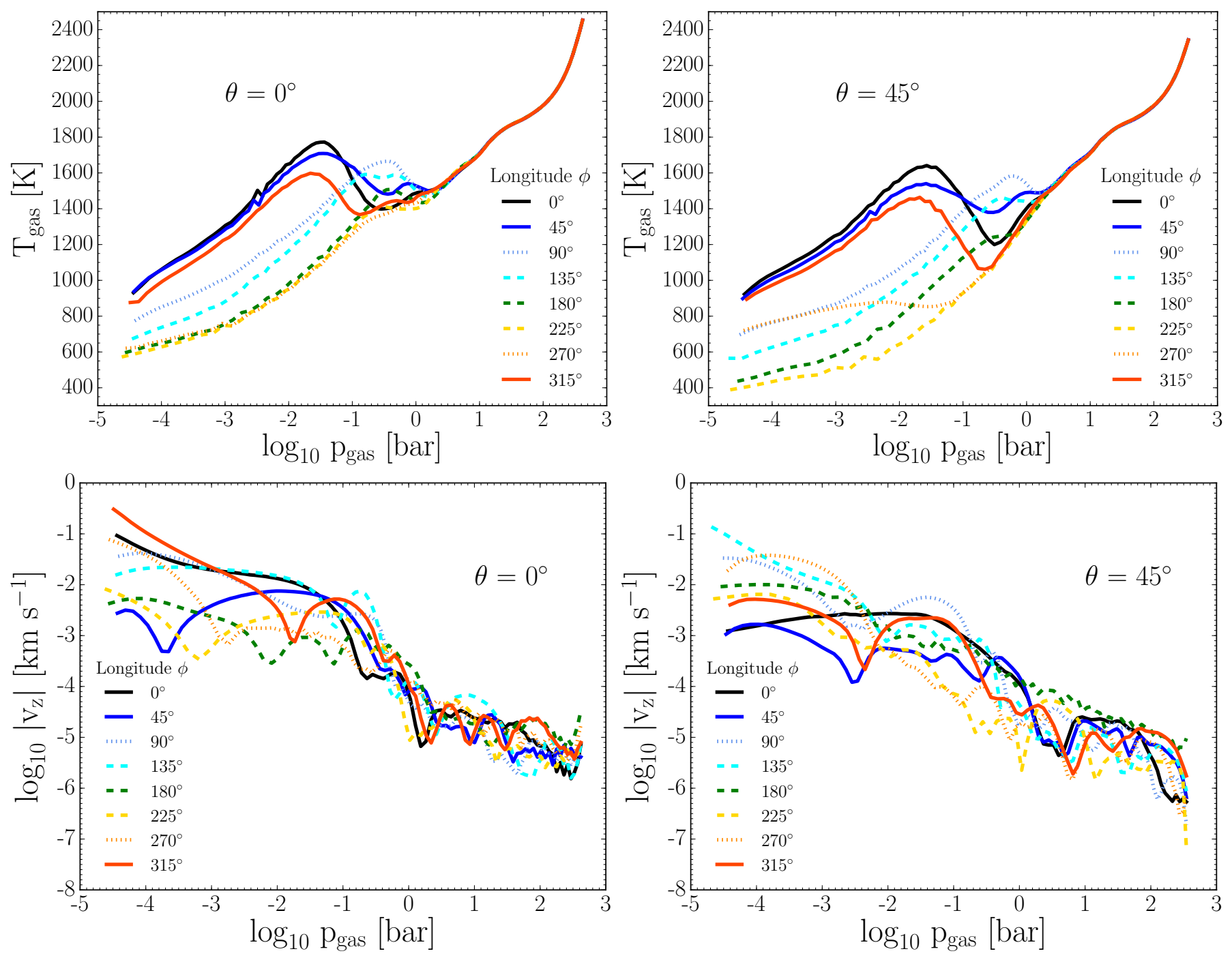

Fig. 2. 1D trajectory results from the 3D RHD HD 189733b atmosphere simulation used as input for the cloud formation model. Top row: $\left(T_{\text {gas }}\right.$, $\left.p_{\text {gas }}\right)$ profiles in steps of longitude $\Delta \phi=+45^{\circ}$ at equatorial, $\theta=0^{\circ}$ and latitude, $\theta=45^{\circ}$ profiles. Bottom row: smoothed vertical gas velocities $\boldsymbol{v}_{\mathrm{z}}\left[\mathrm{km} \mathrm{s}^{-1}\right]$ in steps of longitude $\Delta \phi=+45^{\circ}$ at equatorial, $\theta=0^{\circ}$ and latitude, $\theta=45^{\circ}$ profiles. Both latitudes show a temperature inversion on the dayside. The sub-stellar point is at $\phi=0^{\circ}, \theta=0^{\circ}$. Solid, dotted and dashed lines indicate dayside, day-night terminator and nightside profiles respectively.

the mixing timescale in low mass stellar atmospheres as the time for convective motions $v_{\text {conv }}$ to travel a fraction of the pressure scale height, $H_{\mathrm{p}}(\boldsymbol{r})$

$\tau_{\text {mix,conv }}(\boldsymbol{r})=$ const. $\cdot \frac{H_{\mathrm{p}}(\boldsymbol{r})}{v_{\text {conv }}(\boldsymbol{r})}$.

Helling et al. (2008b), Witte et al. (2009, 2011) represent values for $v_{\text {conv }}(\boldsymbol{r})$, in their 1D DRIFT-PHOENIX model atmospheres, above the convective zone (defined by the Schwarzschild criterion) from inertially driven overshooting of ascending gas bubbles.

\subsubsection{Previous hot Jupiter approach}

On hot Jupiters, in contrast to Earth, Jupiter and brown dwarf atmospheres, the intensity of stellar irradiation can suppress convective motions down to very large pressures (Barman et al. 2001). Therefore turbulent diffusion is likely the dominant vertical transport mechanism. The use of the 3D RHD model results allows us to apply the local vertical velocity component, $\boldsymbol{v}_{\mathrm{z}}(\boldsymbol{r})$, in our cloud formation model. No additional assumptions are required. Hence, $v_{\mathrm{z}}(\boldsymbol{r})$ is known for each atmospheric trajectory chosen, as visualised in Fig. 2.

Some chemical models of hot Jupiters (e.g. Moses et al. 2011) use an approximation of the vertical diffusion coefficient $\boldsymbol{K}_{\mathrm{zz}}(\boldsymbol{r})\left[\mathrm{cm}^{2} \mathrm{~s}^{-1}\right]$ of the gaseous state.

$\boldsymbol{K}_{\mathrm{zz}}(\boldsymbol{r})=H_{\mathrm{p}}(\boldsymbol{r}) \cdot v_{\mathrm{z}}(\boldsymbol{r})$.

The diffusion timescale is then

$\tau_{\text {mix,diff }}(\boldsymbol{r})=$ const. $\cdot \frac{H_{\mathrm{p}}^{2}(\boldsymbol{r})}{K_{\mathrm{zz}}(\boldsymbol{r})}$.

Moses et al. (2011) apply the root-mean-square (rms) vertical velocities derived from global horizontal averages at each atmospheric layer to their chemical models. This results in a horizontally homogenous $K_{\mathrm{zz}}(\boldsymbol{r})$ mixing parameter. Parmentier et al. (2013) derive a global mean value for $K_{\mathrm{zz}}(\boldsymbol{r})$ as a function of local pressure using a passive tracer in their global circulation model (GCM) of HD $189733 \mathrm{~b}$ resulting in $K_{\mathrm{zZ}}(\boldsymbol{r})=10^{7} \mathrm{p}^{-0.65}(\mathrm{p}$ [bar]). Using a global mean smooths 
away all horizontally dependent vertical velocity variations. Any parameterisation of the vertical mixing will depend on the details of the underlying hydrodynamical structure. Parmentier et al. (2013) use a 3D primitive equation model where vertical hydrostatic equilibrium is assumed. The radiation hydrodynamic simulations performed by Dobbs-Dixon \& Agol (2013), applied in this paper, solve the full Navier-Stokes equations and will produce larger vertical velocities compared to the primitive equations. In summary, vertical velocity may be substantially damped in models using the primitive equations. Both Eq. (6) and Parmentier et al. (2013) definitions for $K_{\mathrm{zz}}(\boldsymbol{r})$ assume that the dominant mixing occurs in the vertical direction, possible mixing from horizontal flows are neglected. The time-scale comparison in Sect. 2.4.4 supports this assumption for cloud formation processes. It is worthwhile noting that the idea of diffusive mixing originates from shallow water approximations as applied in solar system research where a 2D velocity field produces shear which creates a turbulent velocity component. Hartogh et al. (2005) outline how local wind shear and the hydrostatic gas pressure are used to represent a vertical mixing.

Some arguments reinforce why vertical mixing is important:

- Regions with low vertical velocity can be replenished of elements by the horizontal winds from high vertical velocity regions in a $3 \mathrm{D}$ situation.

- Large Hadley cell circulation is present and here vertical velocities can be significant and element replenishment to the upper atmosphere may be more efficient.

- Vertical transport is a key mixing process on Earth which has been successfully applied to hot Jupiter chemical models (Moses et al. 2011; Venot et al. 2012; Agúndez et al. 2014).

We use Eq. (7) (const. = 1) as the 1D mixing timescale input for our kinetic dust formation model, with Eq. (6) as the definition of the diffusion coefficient. We adopt the local vertical velocities that result from the Dobbs-Dixon \& Agol (2013) 3D RHD atmosphere simulations for HD $189733 \mathrm{~b}$ as the values for $v_{\mathrm{z}}(\boldsymbol{r})$ (Fig. 2). A 3-point boxcar smoothing was applied to these velocities to reduce the effects of unresolved turbulence. The longitude dependent $K_{\mathrm{zz}}(\phi, r)$ for latitudes $\theta=0^{\circ}, 45^{\circ}$ is shown in Fig. 3 (first row) and the resulting vertical mixing timescales (second row). We include the $K_{\mathrm{zz}}$ relation from Parmentier et al. (2013) in Fig. 3 (dash-dotted line) for comparison. Their linear fit is approximately one order of magnitude lower which is similar to the difference found in HD 189733b and HD 209458b chemical models (Agúndez et al. 2014) who compared the two $K_{\mathrm{zz}}(\boldsymbol{r})$ expressions for Showman et al. (2009) GCM simulations. The current approach aims to capture the unique vertical mixing and thermodynamic conditions at each trajectory, while also accounting for practical modelling of atmospheric mixing.

\subsubsection{Advective timescales}

An important timescale to consider is the charcteristic advective timescale which is a representative timescale for heat to redistribute over the circumference of the globe. The advective timescale is given by

$\tau_{\text {adv }}(\boldsymbol{r})=\frac{\pi r}{v_{\text {horiz }}(\boldsymbol{r})}$,

where $r(z)$ is the radial height of the planet and $v_{\text {horiz }}(\boldsymbol{r})$ the local gas velocity in the horizontal direction $(\phi)$. This timescale gives an idea of how fast thermodynamic conditions can change in the longitudinal direction at a particular height $z$ in the atmosphere. Figure 3 compares the advective timescale to the vertical diffusion timescale (Eq. (7)) and find that both time scales are of the same order of magnitude. This suggests that the convective/turbulent diffusion of upward gaseous material as described in Sect. 2.4.1 occurs approximately at the same timescales as gas advected across the globe. This suggests that, to a first approximation, the 1D ( $\left.T_{\text {gas }}, p_{\text {gas }}\right)$ trajectories used as input for the cloud formation model do not fluctuate rapidly enough to substantially influence the cloud properties.

\subsubsection{Cloud formation timescales}

We compare the cloud particle settling, growth and nucleation timescales that result from our cloud model (Sect. 3) to the mixing and advection timescales that are derived from the hydrodynamic fluid field. The nucleation timescale $\tau_{\text {nuc }}$ is defined as

$\tau_{\text {nuc }}=\frac{n_{\mathrm{d}}}{J_{\star}}$,

the growth timescale $\tau_{\mathrm{gr}}$ by

$\tau_{\mathrm{gr}}=\frac{\sqrt[3]{36 \pi}\langle a\rangle}{3\left|\chi^{\mathrm{net}}\right|}$

and the cloud particle settling timescale $\tau_{\text {setl }}$ by

$\tau_{\text {setl }}=\frac{H_{\mathrm{p}}}{\left|\left\langle v_{\mathrm{dr}}\right\rangle\right|}$

where $\left\langle v_{\mathrm{dr}}\right\rangle$ is the large Knudsen number frictional regime $(K n \gg 1)$ mean drift velocity (Woitke \& Helling 2003, Eq. (63)). Figure 4 shows the timescales at the sub-stellar and anti-stellar points. Our results agree with earlier timescale analysis in Woitke \& Helling (2003) who point out an hierarchical dominance of the cloud formation processes through the atmosphere. In the upper atmosphere nucleation is the most efficient process:

$\tau_{\text {nuc }} \lesssim \tau_{\text {gr }} \ll \tau_{\text {mix }} \sim \tau_{\text {adv }} \ll \tau_{\text {setl }}$.

Deeper in the atmosphere nucleation eventually becomes inefficient and all timescales become comparable

$\tau_{\text {gr }} \lesssim \tau_{\text {mix }} \sim \tau_{\text {adv }} \lesssim \tau_{\text {setl }}$.

The chemical processes that determine the cloud particle formation occur on shorter timescales than the large scale hydrodynamical timescales. This emphasises that the cloud particle formation is a local process.

\subsection{Dust opacity}

Based on the results of the spatially varying cloud properties we calculate the opacity of the cloud particles. We determine the cloud particle absorption and scattering coefficients in each atmospheric layer for wavelengths $0.3 \mu \mathrm{m}, 0.6 \mu \mathrm{m}, 1.1 \mu \mathrm{m}$, $1.6 \mu \mathrm{m}, 3.6 \mu \mathrm{m}, 4.5 \mu \mathrm{m}, 5.8 \mu \mathrm{m}, 8.0 \mu \mathrm{m}, 24.0 \mu \mathrm{m}$, corresponding to the Hubble and Spitzer average band passes. Since the cloud particles are made of mixed solids, the effective $(n, k)$ optical constants is calculated for each particle using effective medium theory. We follow the approach of Bruggeman (1935) where

$\sum_{s}\left(\frac{V_{s}}{V_{\mathrm{tot}}}\right) \frac{\epsilon_{s}-\epsilon_{\mathrm{av}}}{\epsilon_{s}+2 \epsilon_{\mathrm{av}}}=0$,

where $V_{s} / V_{\text {tot }}$ is the volume fraction of solid species $s, \epsilon_{s}$ the dielectric function of solid species $s$ and $\epsilon_{\text {av }}$ the average dielectric function over the total cloud particle volume. The average 

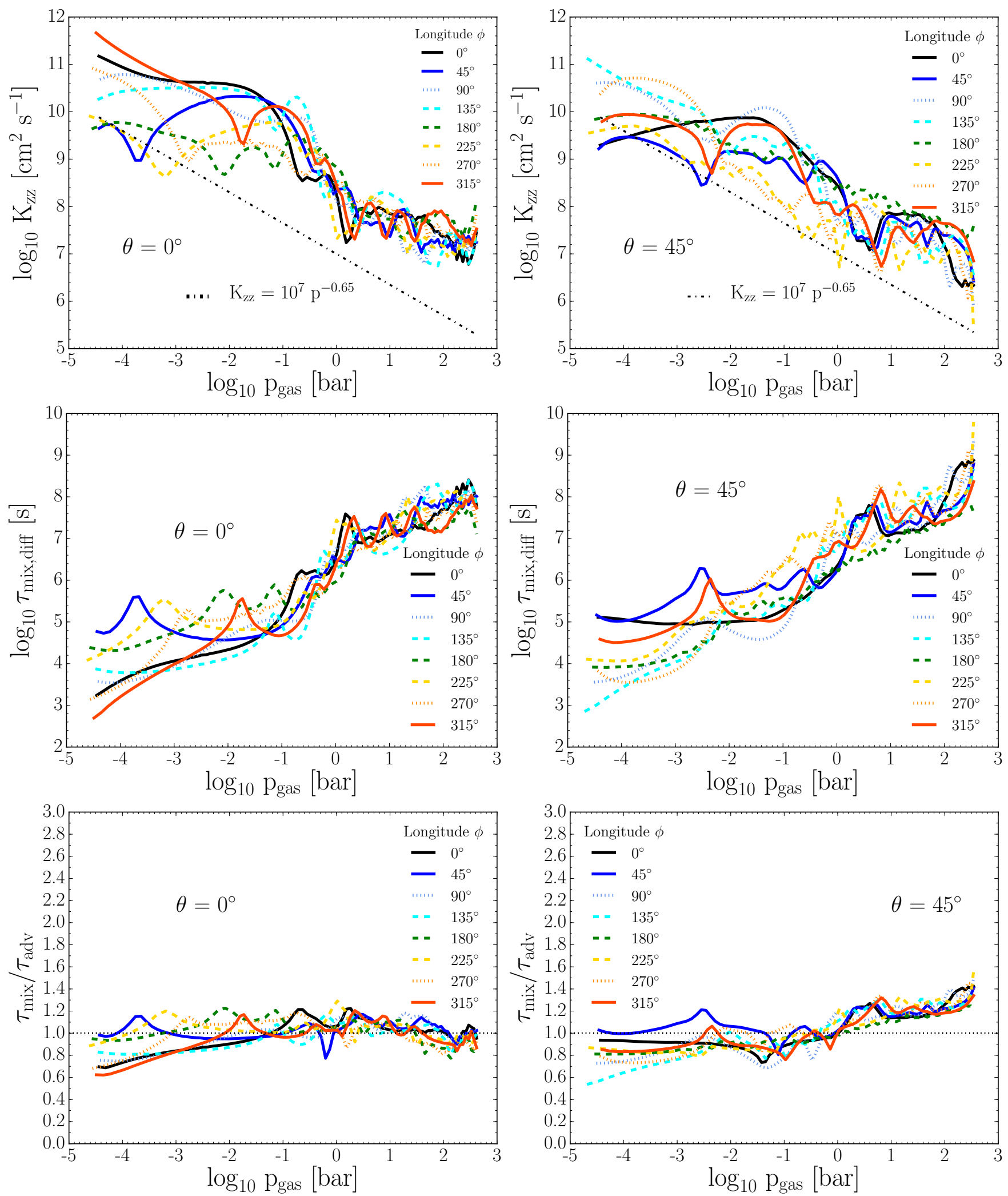

Fig. 3. Top row: vertical diffusion coefficient $K_{\mathrm{zz}}(\boldsymbol{r})=H_{\mathrm{p}} \cdot v_{\mathrm{z}}(\boldsymbol{r})\left[\mathrm{cm}^{2} \mathrm{~s}^{-1}\right]$ applying the smoothed vertical velocities of the radiative-hydrodynamic HD 189733b model at latitudes $\theta=0^{\circ}$ and $\theta=45^{\circ}$, as a function of pressure at $\Delta \phi=+45^{\circ}$ longitude intervals. The $K_{\mathrm{zz}}(\boldsymbol{r})=10^{7} p^{-0.65}(p$ [bar] $)$ expression from Parmentier et al. (2013) is shown as dash-dotted lines. Middle row: the vertical mixing timescales $\tau_{\text {mix,diff }}$ [s] derived from the HD $189733 \mathrm{~b}$ radiative-hydrodynamic model results at latitudes $\theta=0^{\circ}, \theta=45^{\circ}$, as a function of pressure at $\Delta \phi=+45^{\circ}$ longitude intervals. Bottom row: ratio of the mixing and advective timescales at $\theta=0^{\circ}, 45^{\circ}$ respectively. The ratio of the timescales stays approximately equal at all pressures. Solid, dotted and dashed lines indicate dayside, day-night terminator and nightside profiles respectively. 

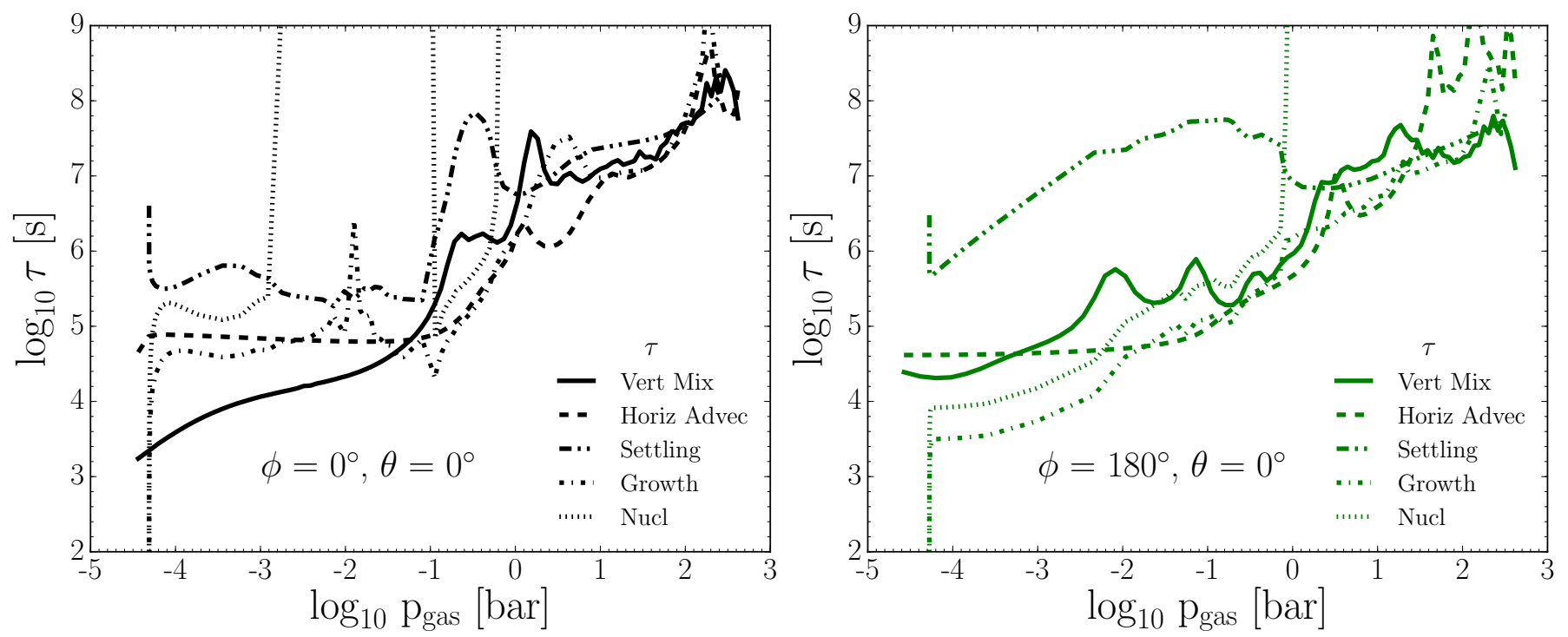

Fig. 4. Vertical mixing, horizontal advection, cloud settling, cloud growth and cloud nucleation timescales at the sub-stellar point (Left) and $\phi=180^{\circ}, \theta=0^{\circ}($ Right $)$.

dielectric function is then found by Newton-Raphson minimisation of Eq. (12). The scattering and extinction cross sections are then calculated using Mie theory for spherical particles (Mie 1908). We follow the approach of Bohren \& Huffman (1983) where the scattering and extinction cross sections are defined as

$$
\begin{aligned}
& C_{\mathrm{sca}}(\lambda, a)=\frac{2 \pi a^{2}}{x^{2}} \sum_{n=1}^{\infty}(2 n+1)\left(\left|a_{n}\right|^{2}+\left|b_{n}\right|^{2}\right), \\
& C_{\mathrm{ext}}(\lambda, a)=\frac{2 \pi a^{2}}{x^{2}} \sum_{n=1}^{\infty}(2 n+1) \operatorname{Re}\left(a_{n}+b_{n}\right),
\end{aligned}
$$

respectively; where $x=2 \pi a / \lambda$ is the wavelength dependent size parameter. The scattering coefficients $a_{\mathrm{n}}$ and $b_{\mathrm{n}}$ are calculated from the material optical $k$ constant (Bohren \& Huffman 1983). The wavelength-dependent absorption and scattering efficiency of a cloud particle is then

$$
\begin{aligned}
& Q_{\mathrm{sca}}(\lambda, a)=\frac{C_{\mathrm{sca}}(\lambda, a)}{\pi a^{2}}, \\
& Q_{\mathrm{abs}}(\lambda, a)=\frac{C_{\mathrm{ext}}(\lambda, a)}{\pi a^{2}}-Q_{\mathrm{sca}},
\end{aligned}
$$

respectively. The total absorption and scattering efficiency $\kappa\left[\mathrm{cm}^{2} \mathrm{~g}^{-1}\right]$ can then be derived by multiplying the corresponding efficiencies with the area and occurrence rate of each cloud particle.

$$
\begin{aligned}
& \kappa_{\mathrm{sca}}(\lambda, a)=Q_{\mathrm{sca}}(\lambda, a) \pi a^{2} n_{\mathrm{d}} / \rho_{\mathrm{gas}}, \\
& \kappa_{\mathrm{abs}}(\lambda, a)=Q_{\mathrm{abs}}(\lambda, a) \pi a^{2} n_{\mathrm{d}} / \rho_{\mathrm{gas}} .
\end{aligned}
$$

We include all 12 solid dust species in the opacity calculations. References for their optical constants are presented in Table 2. We assume that for all Mie calculations the grain size is given by the mean grain radius $\langle a\rangle$ (Eq. (4)) with number density $n_{\mathrm{d}}$ (Eq. (3)) for each atmospheric layer.

\section{Mineral clouds in the atmosphere of HD 189733b}

We apply the dust formation theory developed by Woitke \& Helling (2003, 2004), Helling \& Woitke (2006) and
Table 2. References for the $(n, k)$ optical constants of the 12 different solids.

\begin{tabular}{cc}
\hline \hline Solid species & Source \\
\hline $\mathrm{TiO}_{2}[\mathrm{~s}]$ & Ribarsky in Palik (1985) \\
$\mathrm{Al}_{2} \mathrm{O}_{3}[\mathrm{~s}]$ & Zeidler et al. (2013) \\
$\mathrm{CaTiO}_{3}[\mathrm{~s}]$ & Posch et al. (2003) \\
$\mathrm{Fe}_{2} \mathrm{O}_{3}[\mathrm{~s}]$ & Unpublished \\
$\mathrm{FeS}[\mathrm{s}]$ & Henning et al. (1995) \\
$\mathrm{FeO}[\mathrm{s}]$ & Henning et al. (1995) \\
$\mathrm{Fe}[\mathrm{s}]$ & Posch et al. (2003) \\
$\mathrm{SiO}[\mathrm{s}]$ & Philipp in Palik (1985) \\
$\mathrm{SiO}_{2}[\mathrm{~s}]$ & Posch et al. (2003) \\
$\mathrm{MgO}^{a}[\mathrm{~s}]$ & Palik (1985) \\
$\mathrm{MgSiO}_{3}[\mathrm{~s}]$ & Dorschner et al. (1995) \\
$\mathrm{Mg}_{2} \mathrm{SiO}_{4}[\mathrm{~s}]$ & Jäger et al. (2003) \\
\hline
\end{tabular}

Notes. (a) http://www.astro.uni-jena.de/Laboratory/OCDB/ oxsul.html

Helling et al. (2008b) in its 1D stationary form to 1D output trajectories of a 3D RHD model of HD 189733b, as described in Dobbs-Dixon \& Agol (2013). In the following, we present local and global cloud structures for HD $189733 \mathrm{~b}$ and discuss detailed results on cloud properties such as grain sizes, material composition, element abundances, dust-to-gas ratio and $\mathrm{C} / \mathrm{O}$ ratio. We investigate general trends of the cloud structure as it varies throughout the atmosphere and make first attempts to study the cloud properties across the planetary globe.

\subsection{The cloud structure of HD 189733b at the sub-stellar point}

The substellar point $\left(\phi=0^{\circ}, \theta=0^{\circ}\right)$ is the most directly irradiated point in atmospheres of hot Jupiters such as HD 189733b which is measured by observing before, during and after secondary transit (occulatation). We use this well defined location to provide a detailed description of the vertical cloud structure. We compare this atmospheric trajectory to other longitudes in Sect. 3.2 and to other latitudes in Sect. 3.3. Figure 5 shows that the cloud structure starts with the formation of seed particles 

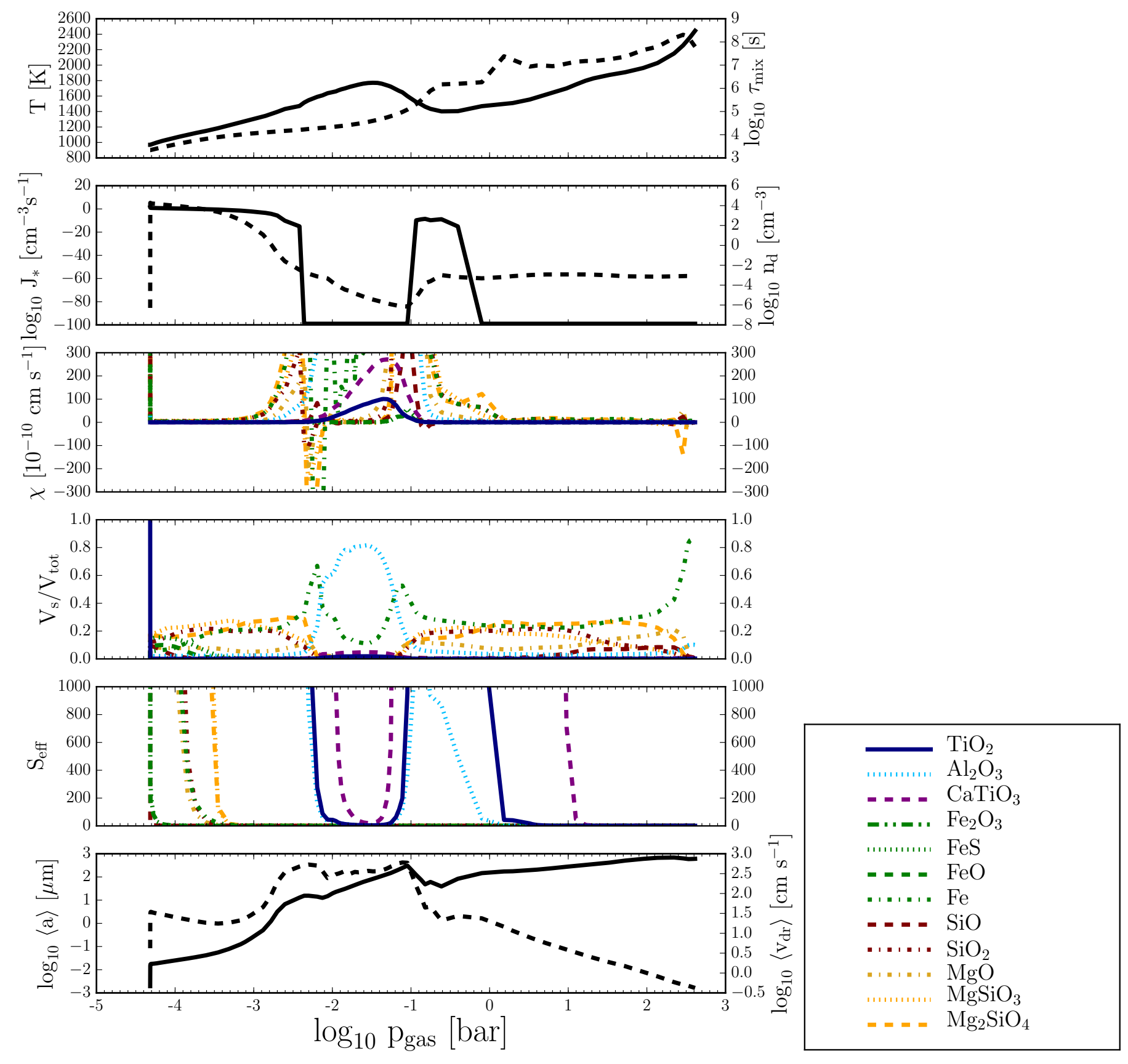

Fig. 5. HD 189733b's calculated cloud structure at the sub-stellar point $\left(\theta=0^{\circ}, \phi=0^{\circ}\right)$. Left: 1 st panel: local gas temperature $T_{\text {gas }}[\mathrm{K}]$ (solid, left) and mixing timescale $\tau_{\text {mix }}$ [s] (dashed, right). 2nd panel: nucleation rate $J_{*}\left[\mathrm{~cm}^{-3} \mathrm{~s}^{-1}\right]$ (solid, left) and dust number density $n_{\mathrm{d}}\left[\mathrm{cm}^{-3}\right]($ dashed, right). 3rd panel: growth velocity of material $s \chi\left[\mathrm{cm} \mathrm{s}^{-1}\right] .4$ th panel: volume fraction $V_{s} / V_{\text {tot }}$ of solid $s$. 5th panel: effective supersaturation ratio $S_{\text {eff }}$ of individual solids. 6th panel: cloud particle mean radius $\langle a\rangle[\mu \mathrm{m}]$ (solid, left) and mean drift velocity $\left\langle v_{\mathrm{dr}}\right\rangle$ [cm s $\left.{ }^{-1}\right]$ (dashed, right). Right: key showing line-style and colour of our considered dust species.

$\left(J_{*}\left[\mathrm{~cm}^{-3} \mathrm{~s}^{-1}\right]-\right.$ nucleation rate) occurring at the upper pressure boundary of $\sim 10^{-4}$ bar. After the first surface growth processes occur on the seed particles, the cloud particles then gravitationally settle into the atmospheric regions below (towards higher density/pressure). Primary nucleation is efficient down to $\sim 10^{-2.5}$ bar where it drops off significantly, indicating that the local temperature is too high for further nucleation and that the seed forming species has been substantially depleted. The gas-grain surface chemical reactions that form the grain mantle (Eq. (2)) increase in rate as the cloud particles fall inward. This is due to the cloud particles encountering increasing local gas density, and therefore more condensible material is available to react with cloud particles. This surface growth becomes more efficient from $\sim 10^{-3} \ldots \sim 10^{-2}$ bar until the local temperature is so hot that the materials become thermally unstable and evaporate. The evaporation region results in a half magnitude decrease of the cloud particle sizes (negative $\chi$ ) in the center region of the cloud. The relative volume fractions of the solid " $s$ ", $V_{s} / V_{\text {tot }}$, represents the material composition of the cloud particles. The cloud particle composition is dominated by silicates and oxides such as $\mathrm{MgSiO}_{3}[\mathrm{~s}](\sim 27 \%), \mathrm{Mg}_{2} \mathrm{SiO}_{4}[\mathrm{~s}](\sim 20 \%)$, $\mathrm{SiO}_{2}[\mathrm{~s}](\sim 21 \%)$ at the upper regions $\lesssim 10^{-2.5}$ bar. Fe[s] contributes $\lesssim 20 \%$ to the volume of the cloud particle in this region. The other 8 growth species $\left(\mathrm{TiO}_{2}[\mathrm{~s}](\sim 0.03 \%), \mathrm{Al}_{2} \mathrm{O}_{3}[\mathrm{~s}](\sim 2 \%)\right.$, 
Table 3. Volume fraction $V_{\mathrm{s}} / V_{\text {tot }}[\%]$ for the 12 solid species included in kinetic the cloud model.

\begin{tabular}{|c|c|c|c|c|c|c|c|}
\hline Pressure [bar] & $10^{-4}$ & $10^{-3}$ & $10^{-2}$ & $10^{-1}$ & 1 & 10 & Cloud base \\
\hline \multirow{2}{*}{$\langle a\rangle[\mu \mathrm{m}]$} & 0.025 & 0.23 & 19.9 & 307 & 146 & 275 & 605 \\
\hline & 0.018 & 0.035 & 0.15 & 12.3 & 174 & 338 & 1088 \\
\hline \multicolumn{8}{|l|}{ Solid species } \\
\hline \multirow{2}{*}{$\mathrm{TiO}_{2}[\mathrm{~s}]$} & 0.03 & 0.03 & 1.22 & 0.26 & 0.05 & 0.02 & 0.22 \\
\hline & 0.06 & 0.04 & 0.03 & 0.03 & 0.02 & 0.01 & 0.24 \\
\hline \multirow{2}{*}{$\mathrm{Al}_{2} \mathrm{O}_{3}[\mathrm{~s}]$} & 2.11 & 2.43 & 60.98 & 13.99 & 3.68 & 2.84 & 10.03 \\
\hline & 2.06 & 2.06 & 2.13 & 2.42 & 2.44 & 2.49 & 10.03 \\
\hline \multirow{2}{*}{$\mathrm{CaTiO}_{3}[\mathrm{~s}]$} & 0.13 & 0.16 & 3.56 & 0.87 & 0.24 & 0.22 & 0.64 \\
\hline & 0.07 & 0.10 & 0.14 & 0.17 & 0.17 & 0.20 & 0.77 \\
\hline \multirow{2}{*}{$\mathrm{Fe}_{2} \mathrm{O}_{3}[\mathrm{~s}]$} & 0.10 & $>0.01$ & $>0.01$ & $>0.01$ & $>0.01$ & $>0.01$ & $>0.01$ \\
\hline & 9.69 & 9.68 & 0.07 & $>0.01$ & $>0.01$ & $>0.01$ & $>0.01$ \\
\hline \multirow{2}{*}{$\mathrm{FeS}[\mathrm{s}]$} & 14.44 & 0.22 & 0.03 & 0.09 & 0.05 & 0.02 & 0.03 \\
\hline & 12.12 & 12.12 & 14.45 & 0.10 & 0.05 & 0.02 & 0.03 \\
\hline \multirow{2}{*}{$\mathrm{FeO}[\mathrm{s}]$} & 8.87 & 0.06 & 0.03 & 0.07 & 0.02 & 0.02 & 0.09 \\
\hline & 7.63 & 7.63 & 7.93 & 0.03 & 0.02 & 0.02 & 0.09 \\
\hline \multirow{2}{*}{$\mathrm{Fe}[\mathrm{s}]$} & 7.87 & 21.08 & 30.93 & 45.00 & 24.29 & 22.49 & 87.15 \\
\hline & 4.52 & 4.52 & 8.50 & 21.14 & 21.29 & 21.75 & 86.80 \\
\hline \multirow{2}{*}{$\mathrm{SiO}[\mathrm{s}]$} & 2.42 & 0.06 & 0.08 & 0.70 & 0.48 & 3.82 & 0.92 \\
\hline & 8.84 & 9.03 & 1.12 & 0.11 & 0.44 & 5.20 & 0.99 \\
\hline \multirow{2}{*}{$\mathrm{SiO}_{2}[\mathrm{~s}]$} & 17.90 & 20.08 & 0.75 & 7.88 & 19.94 & 20.60 & 0.12 \\
\hline & 9.92 & 10.13 & 19.35 & 21.26 & 22.18 & 20.33 & 0.13 \\
\hline \multirow{2}{*}{$\mathrm{MgO}[\mathrm{s}]$} & 7.73 & 5.13 & 1.97 & 11.10 & 8.05 & 8.87 & 0.79 \\
\hline & 7.21 & 7.28 & 7.61 & 5.40 & 6.51 & 9.35 & 0.89 \\
\hline \multirow{2}{*}{$\mathrm{MgSiO}_{3}[\mathrm{~s}]$} & 22.47 & 24.11 & 0.14 & 8.96 & 20.77 & 15.79 & $>0.01$ \\
\hline & 21.74 & 20.71 & 22.41 & 22.02 & 21.82 & 15.25 & $>0.01$ \\
\hline \multirow{2}{*}{$\mathrm{Mg}_{2} \mathrm{SiO}_{4}[\mathrm{~s}]$} & 15.93 & 26.64 & 0.30 & 11.09 & 21.41 & 25.31 & 0.02 \\
\hline & 16.15 & 16.70 & 16.23 & 27.31 & 26.59 & 25.39 & 0.04 \\
\hline
\end{tabular}

Notes. The first row of each species corresponds to the sub-stellar trajectory $\left(\phi=0^{\circ}, \theta=0^{\circ}\right)$ cloud structure. The second row corresponds to the nightside trajectory $\phi=180^{\circ}, \theta=0^{\circ}$ cloud structure. Note: the pressure at the cloud base is different for the two profiles.

$\mathrm{CaTiO}_{3}[\mathrm{~s}](\sim 0.15 \%), \quad \mathrm{Fe}_{2} \mathrm{O}_{3}[\mathrm{~s}](\sim 0.001 \%), \quad \mathrm{FeS}[\mathrm{s}](\sim 1.6 \%)$, $\mathrm{FeO}[\mathrm{s}](\sim 0.35 \%), \quad \mathrm{SiO}[\mathrm{s}](\sim 0.05 \%), \quad \mathrm{MgO}[\mathrm{s}](\sim 7 \%)) \quad$ constitute the remaining grain volume. An evaporation region at $\sim 10^{-2.5}$ bar before the temperature maximum at $\sim 10^{-1.5}$ bar alters the grain composition dramatically. $\mathrm{Al}_{2} \mathrm{O}_{3}[\mathrm{~s}]$ and $\mathrm{Fe}[\mathrm{s}]$ dominate the grain composition in this region as the less stable silicates and oxides have evaporated from the grain surface. At the temperature maximum the grain is composed of $\mathrm{Al}_{2} \mathrm{O}_{3}[\mathrm{~s}](\sim 80 \%)$ and $\mathrm{Fe}[\mathrm{s}](\sim 15 \%)$. This suggests the presence of a cloud section more transparent than surrounding layers (Fig. 12). We address the issue of transparency in Sect. 5. Deeper in the atmosphere, a temperature inversion occurs (Fig. 2) starting from $\sim 10^{-1.5}$ bar. The temperature drops by $\sim 400 \mathrm{~K}$ from $\sim 10^{-1.5} \ldots \sim 10^{-0.5}$ bar. This temperature decrease allows a secondary nucleation region from $\sim 10^{-1} \ldots 1$ bar. The number density of grains jumps by $\sim 2$ orders of magnitude at the secondary nucleation region, as a result of formation of many new cloud particles. This causes a dip in the mean grain size at $\sim 10^{-1}$ bar. Such secondary nucleation has not been seen in our cloud model results in brown dwarf nor non-irradiated giant gas planet atmospheres. Silicates and oxides are now thermally stable and once again form the grain mantle. The grain composition is approximately a 70-20-10\% mix of silicates and oxides, iron and other material respectively in this region, similar to the composition before the temperature maximum. At the cloud base, $\mathrm{Fe}[\mathrm{s}]$ dominates the composition $(\sim 35 \%)$ with $\mathrm{MgO}[\mathrm{s}]$ and $\mathrm{Mg}_{2} \mathrm{SiO}_{4}[\mathrm{~s}]$ making up $\sim 16 \%$ and $\sim 23 \%$ respectively. Table 3 shows the percentage volume fraction $V_{\mathrm{s}} / V_{\text {tot }}$ of the 12 dust species at the sub-stellar point and the $\phi=180^{\circ}, \theta=0^{\circ}$ trajectory at $10^{-4}, 10^{-3}, 10^{-2}, 10^{-1}, 1$ and 10 bar.
Our results show that the entire vertical atmospheric range considered here $\left(\sim 10^{-4.5} \ldots 10^{3}\right.$ bar $)$ is filled with dust. The exact properties of this dust such as size, composition and number density change depending on the local thermodynamical state. The 3D RHD model does not expand to such low pressures and densities that the cloud formation processes becomes inefficient (Sect. 5). This suggests that clouds can be present at a higher and lower pressure than the present 3D RHD model boundary conditions allow.

\subsection{Cloud structure changes with longitude (east-west)}

One of the main features of the RHD simulation is the equatorial jet structure which transports heat over the entire $360^{\circ}$ longitude. The presence of this jet changes the thermodynamic structure of the atmosphere which affects the resulting global cloud structure. We sampled the 3D RHD results in longitude steps of $\Delta \phi=+45^{\circ}$ at the equatorial region $\theta=0^{\circ}$ to investigate if cloud properties change significantly from dayside to nightside. Figure 2 shows that the nightside $\left(T_{\text {gas }}, p_{\text {gas }}\right)$ profiles can be $\sim 200 \mathrm{~K}$ cooler than the dayside in the upper atmospheric regions $\left(\sim 10^{-4.5} \ldots 10^{-1}\right.$ bar). Figure 6 shows the nucleation rate $J_{*}\left[\mathrm{~cm}^{-3} \mathrm{~s}^{-1}\right]$ for dayside (solid lines), day-night terminator (dotted lines) and nightside (dashed lines) sample trajectories. The dayside nucleation rates fall off quicker with atmospheric depth compared to the terminator and nightside profiles, particularly relevant for interpreting transit spectra. At longitude $\phi=315^{\circ}$ a secondary nucleation region emerges, similar to the sub-stellar point structure (Fig. 5). The terminator and nightside nucleation regions extend further into the atmosphere to $\sim 10^{-1}$ bar. As a consequence of the different nucleation rates between dayside 
A\&A 580, A12 (2015)
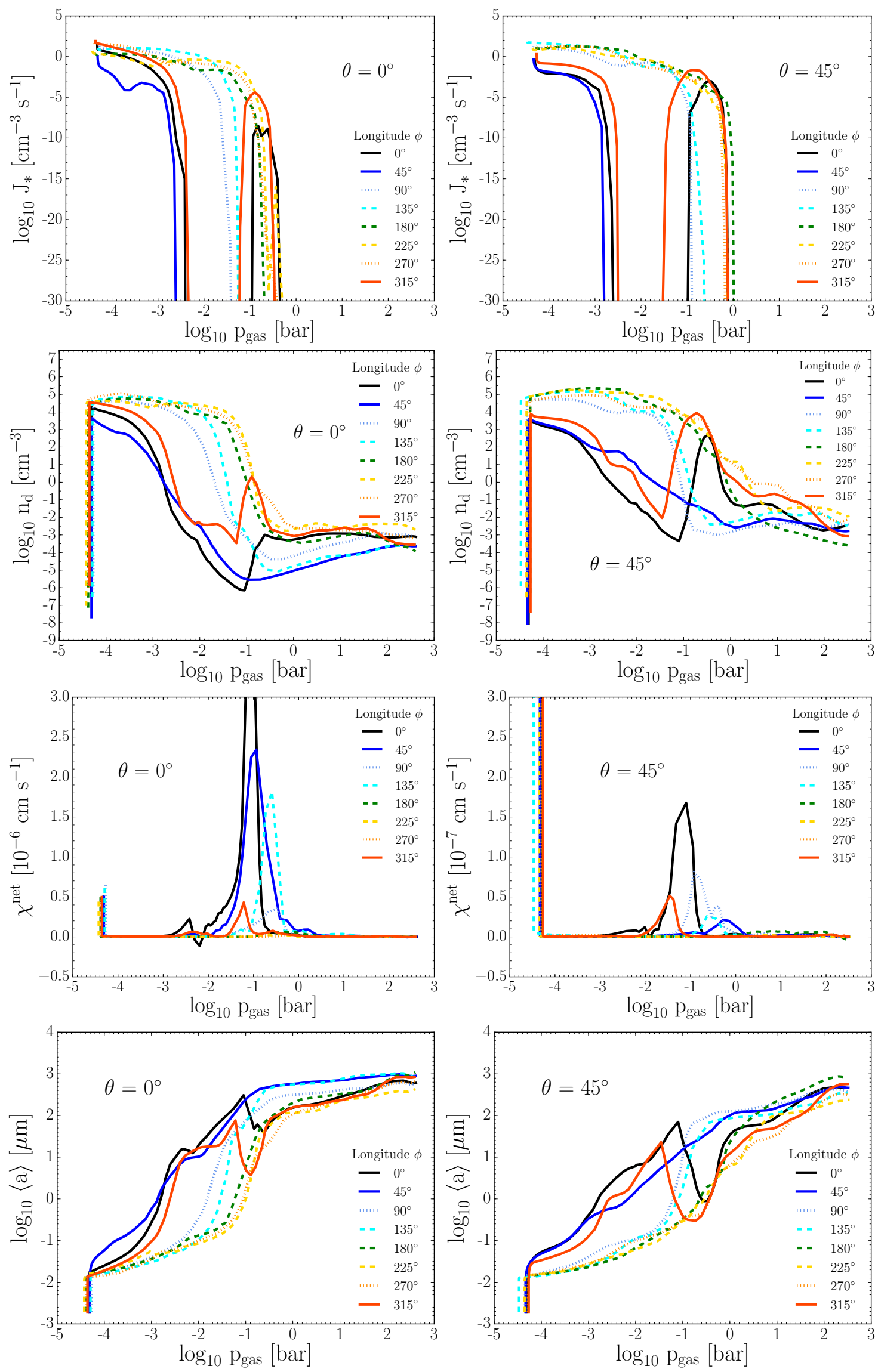

Fig. 6. Dust properties as a function of gas pressure for $\Delta \phi=+45^{\circ}$ longitude intervals for latitudes $\theta=0^{\circ}$ (left column) and $45^{\circ}$ (right column): top row: nucleation rate $J_{*}\left[\mathrm{~cm}^{-3} \mathrm{~s}^{-1}\right]$. Second row: dust number density $n_{\mathrm{d}}\left[\mathrm{cm}^{-3}\right]$. Third row: net dust growth velocity $\chi^{\text {net }}\left[\mathrm{cm} \mathrm{s}^{-1}\right]$. Bottom row: mean grain size $\langle a\rangle[\mu \mathrm{m}]$. Solid, dotted and dashed lines indicate dayside, day-night terminator and nightside profiles respectively. 
E. Lee et al.: Modelling the local and global cloud formation on HD 189733b
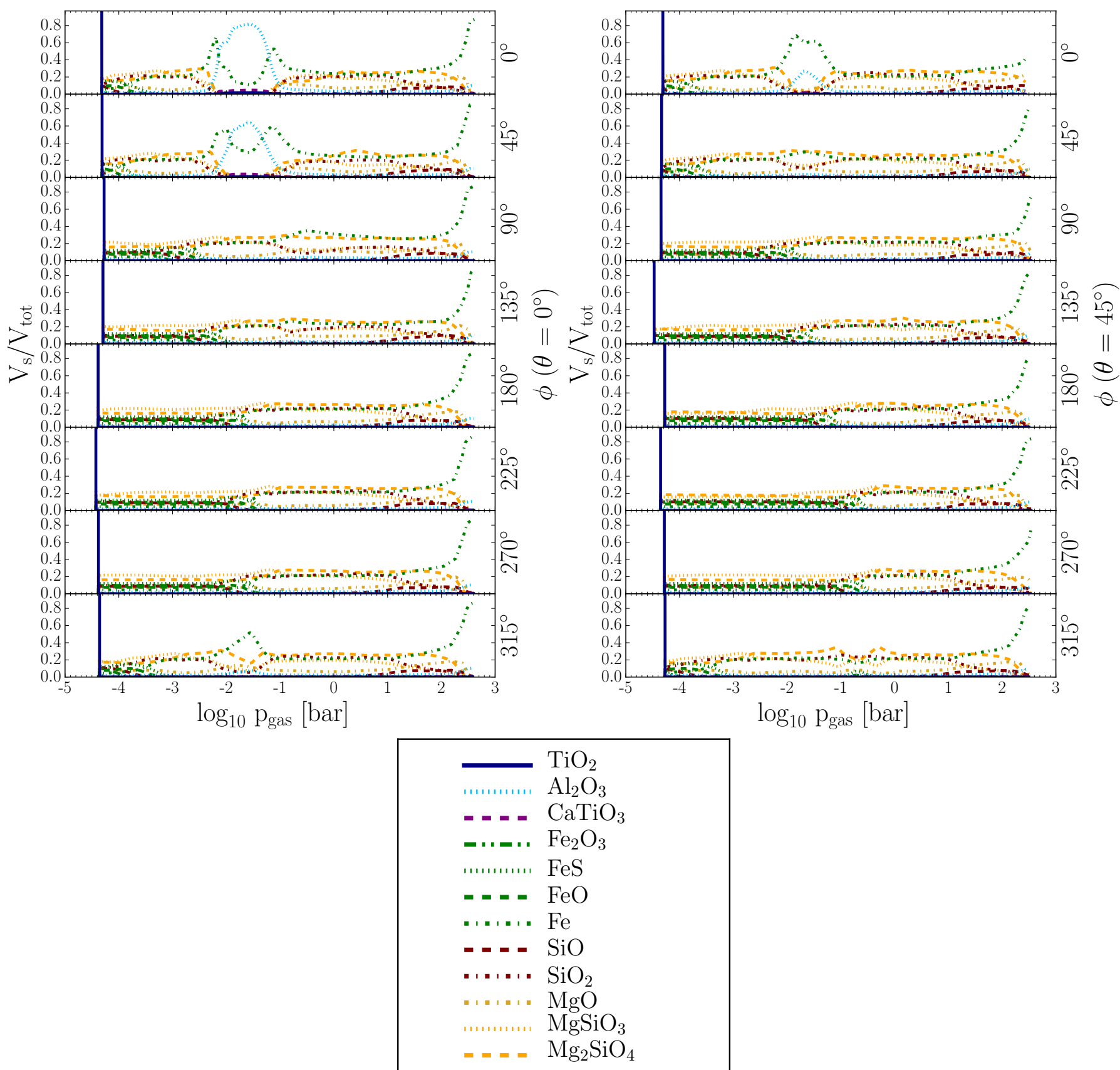

Fig. 7. Top: dust composition volume fraction $V_{s} / V_{\text {tot }}$ as a function of pressure at $\theta=0^{\circ}($ Left $)$ and $\theta=45^{\circ}($ Right $)$ in $\Delta \phi=+45^{\circ}$ longitude intervals. Grain composition is generally dominated by silicate-oxides including $\mathrm{MgSiO}_{3}[\mathrm{~s}]$ apart from localised regions such as $10^{-2} \ldots 10^{-1}$ bar at $\phi=0^{\circ}, 45^{\circ}, \theta=0^{\circ}$. Fe [s] dominates at $10^{-2} \ldots 10^{-1}$ bar at $\phi=0^{\circ}, \theta=45^{\circ}$. Fe[s] grains remain at the cloud base as the most thermally stable element. Bottom: key showing line-style and colour of our considered dust species.

and nightside, the number density of cloud particles $n_{\mathrm{d}}\left[\mathrm{cm}^{-3}\right]$ is greater on the nightside down to pressures of $\sim 10^{-1}$ bar. At this pressure, the secondary nucleation regions on the dayside, $\phi=0^{\circ}, 315^{\circ}$ profiles spike up the number density comparable to values on the nightside. From Fig. 6 the net growth velocity $\chi^{\text {net }}\left[\mathrm{cm} \mathrm{s}^{-1}\right]$ shows that the most efficient growth regions for the grains is approximately $10^{-3} \ldots 1$ bar for the dayside and $10^{-2} \ldots 1$ bar for the nightside. Although the temperature of the local gas phase plays a role in increasing the growth rate, it is the increasing local density of material (as the particle falls thought the atmosphere) that is the dominating factor in determining growth rates (Eq. (2); Fig. 8). Consequently, the mean grain size $\langle a\rangle[\mu \mathrm{m}]$ shows a stronger increase from $\sim 10^{-4} \ldots 10^{-1}$ bar on the dayside than the nightside. The mean grain size dips at $\sim 10^{-1}$ bar for longitudes $\phi=0^{\circ}$ and $315^{\circ}$ due to the increase of grain number density as now the same number of gaseous growth species have to be distributed over a larger surface area. Figure 7 shows the volume fraction $V_{s} / V_{\text {tot }}$ of the solid species " $s$ ". The dust composition is generally dominated by silicates and oxides $(\sim 60 \%)$ such as $\mathrm{MgSiO}_{3}$ [s], $\mathrm{Mg}_{2} \mathrm{SiO}_{4}$ [s] and $\mathrm{SiO}_{2}$ [s] with $(\sim 20 \%) \mathrm{Fe}[\mathrm{s}]$ and $\mathrm{FeO}[\mathrm{s}]$ content. At the cloud base the grain is primarily composed of $\mathrm{Fe}[\mathrm{s}](\sim 85 \%)$ and $\mathrm{Al}_{2} \mathrm{O}_{3}[\mathrm{~s}](\sim 10 \%)$. The $\phi=45^{\circ}, \theta=0^{\circ}$ cloud structure contains an $\mathrm{Al}_{2} \mathrm{O}_{3}[\mathrm{~s}](\sim 60 \%)$ and $\mathrm{Fe}[\mathrm{s}](\sim 30 \%)$ dominant region from $\sim 10^{-2} \ldots 10^{-1}$ bar similar to the sub-stellar point structure. Table 3 summarises the percentage volume fraction $V_{\mathrm{s}} / V_{\text {tot }}$ and the mean cloud particle 

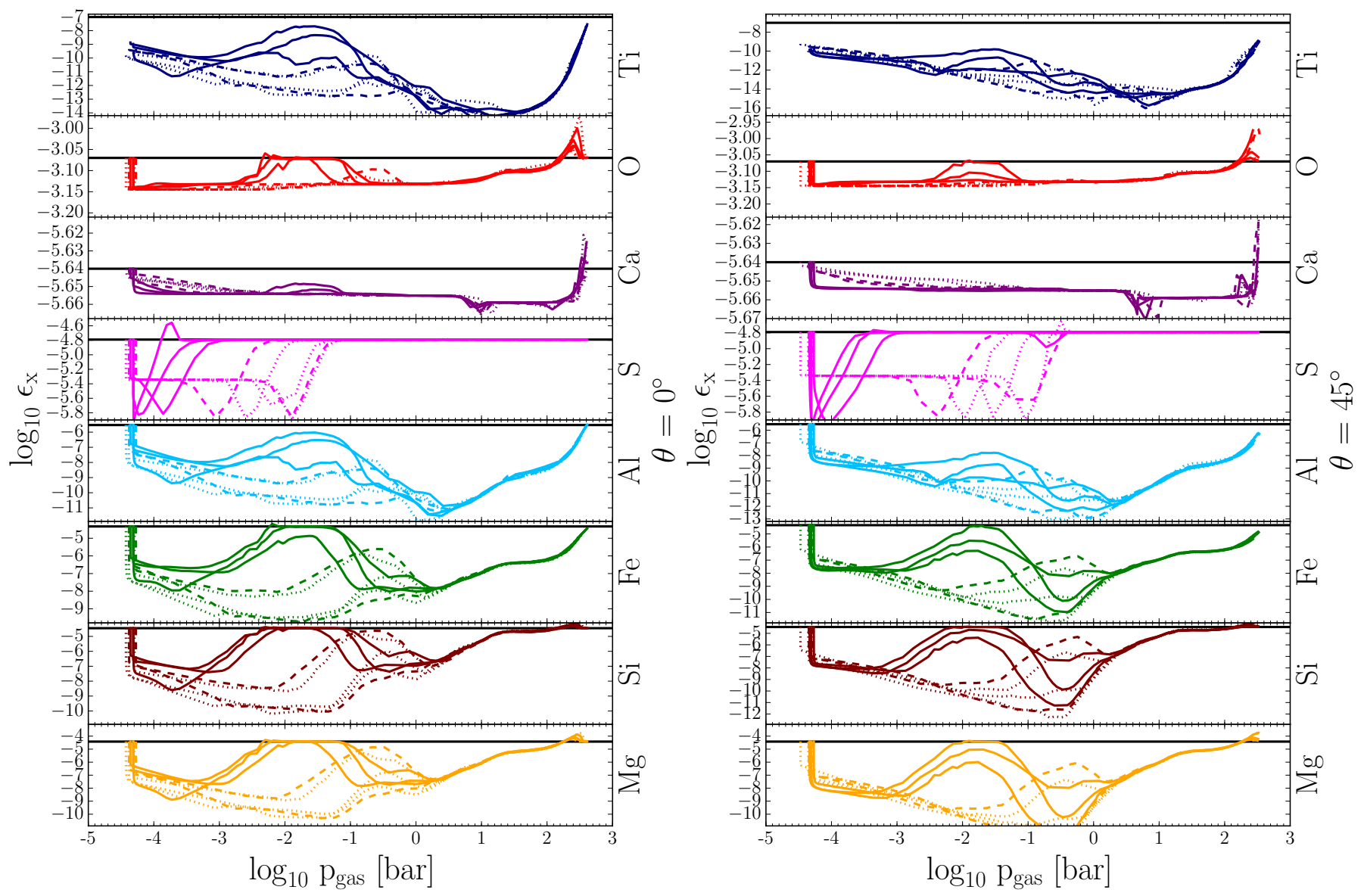

Fig. 8. Gas phase element abundances $\epsilon_{\mathrm{x}}$ as a function of pressure at $\theta=0^{\circ}($ Left $)$ and $\theta=45^{\circ}($ Right $)$ in $\Delta \phi=+45^{\circ}$ longitude intervals. We consider 8 elements: $\mathrm{Mg}$ (orange), Si (maroon), Ti (blue), O (red), Fe (green), Al (cyan), Ca (purple), S (magenta) that constitute the growth species. Horizontal black lines indicate solar abundance $\epsilon_{\mathrm{x}}^{0}$. A decrease in element abundance indicates condensation of growth species onto cloud particle surface. An increase indicates evaporation of molecules constituted of that element from the cloud particle surface. Dayside profiles (solid) are $\phi=0^{\circ}, 45^{\circ}, 315^{\circ}, \theta=0^{\circ}, 45^{\circ}$. Day-night terminator profiles (dashed) are $\phi=90^{\circ}, 270^{\circ}, \theta=0^{\circ}, 45^{\circ}$. Nightside profiles (dotted) are $\phi=135^{\circ}$, $180^{\circ}, 225^{\circ}, \theta=0^{\circ}, 45^{\circ}$.

size $\langle a\rangle$ of the 12 dust species at the sub-stellar point and the $\phi=180^{\circ}, \theta=0^{\circ}$ trajectory at $10^{-4}, 10^{-3}, 10^{-2}, 10^{-1}, 1$ and 10 bar pressures.

\subsection{Cloud structure changes with latitude (north-south)}

At higher latitudes $\theta \gtrsim 40^{\circ}$ the 3D RHD model produces a jet structure flowing in the opposite direction to the equatorial jet at dayside longitudes (Tsai et al. 2014). This significantly alters the $\left(T_{\text {gas }}, p_{\text {gas }}\right)$ and vertical velocity profiles (Fig. 2 ). These latitudes also contain the coldest regions of the nightside where vortexes easily form and dominate the atmosphere dynamics (DobbsDixon \& Agol 2013, Fig. 1). To investigate the cloud structure at these latitudes we repeated our trajectory sampling for latitudes of $\theta=45^{\circ}$ in longitude steps of $\Delta \phi=+45^{\circ}$. Figure 6 shows the nucleation rate $J_{*}\left[\mathrm{~cm}^{-3} \mathrm{~s}^{-1}\right]$ for dayside (solid lines), daynight terminator (dotted lines) and nightside (dashed lines) sample trajectories. The profiles are similar to the equatorial $\theta=0^{\circ}$ regions with double nucleation peaks at $\phi=0^{\circ}$ and $315^{\circ}$. Again, there is an increase in number density $n_{\mathrm{d}}\left[\mathrm{cm}^{-3}\right]$ (Fig. 6) from $10^{-1} \ldots 1$ bar due to second nucleation regions at $\phi=0^{\circ}$ and $315^{\circ}$. The growth velocity $\chi^{\text {net }}$ is generally an order of magnitude lower on the dayside than the equatorial regions. This results in higher latitude clouds containing smaller grain sizes compared to their equatorial counterparts.

\subsection{Element depletion, C/O ratio and dust-to-gas ratio}

The cloud formation process strongly depletes the local gas phase of elements, primarily through extremely efficient solid surface growth processes. We consider the 8 elements that constitute the solid materials of the cloud particles, $\mathrm{Mg}, \mathrm{Si}, \mathrm{Ti}, \mathrm{O}$, $\mathrm{Fe}, \mathrm{Al}, \mathrm{Ca}$ and $\mathrm{S}$ assuming an initial solar element abundance $\left(\epsilon_{\mathrm{x}}^{0}\right)$ for all layers. Figure 8 shows the elemental abundance $\epsilon_{\mathrm{x}}$ (ratio to Hydrogen abundance) of each element as a function of pressure at each of the sample trajectories. Depletion occurs due to the formation of solids made of the these element onto the cloud particle surface and by nucleation of new cloud particles. Increase in element abundances correspond to regions of solid material evaporation. $\mathrm{Ti}$ is depleted at the upper boundary due to immediate efficient nucleation. For dayside profiles $\phi=0^{\circ}$, $45^{\circ}, 315^{\circ}, \theta=0^{\circ}, 45^{\circ}$, from $10^{-4.5} \ldots 10^{-3}$ bar, $\mathrm{Mg}$, Ti, Si, Al and $\mathrm{Fe}$ are depleted by $\sim 1$ order of magnitude while $\mathrm{O}, \mathrm{S}$ and $\mathrm{Ca}$ are depleted by $\sim 10 \%$. These profiles return to initial solar abundance values at $\sim 10^{-2}$ bar where the solid material from the grain surface evaporates, returning elements to the gas phase. O, Fe, $\mathrm{Si} \mathrm{S}$ and $\mathrm{Mg}$ abundance can slightly overshoot solar abundance values at their respective maximums. Elements are most heavily depleted in these profiles at $\sim 10^{-1}$ bar where the most efficient surface growth occurs. $\mathrm{Mg}, \mathrm{Si}$, and Fe are depleted by $\sim 3$ orders of magnitude, Ti by 8 orders of magnitude and Al by 5 orders of magnitude. $\mathrm{O}, \mathrm{S}$ and $\mathrm{Ca}$ are again depleted by $\sim 10 \%$. Fe, Al, S 

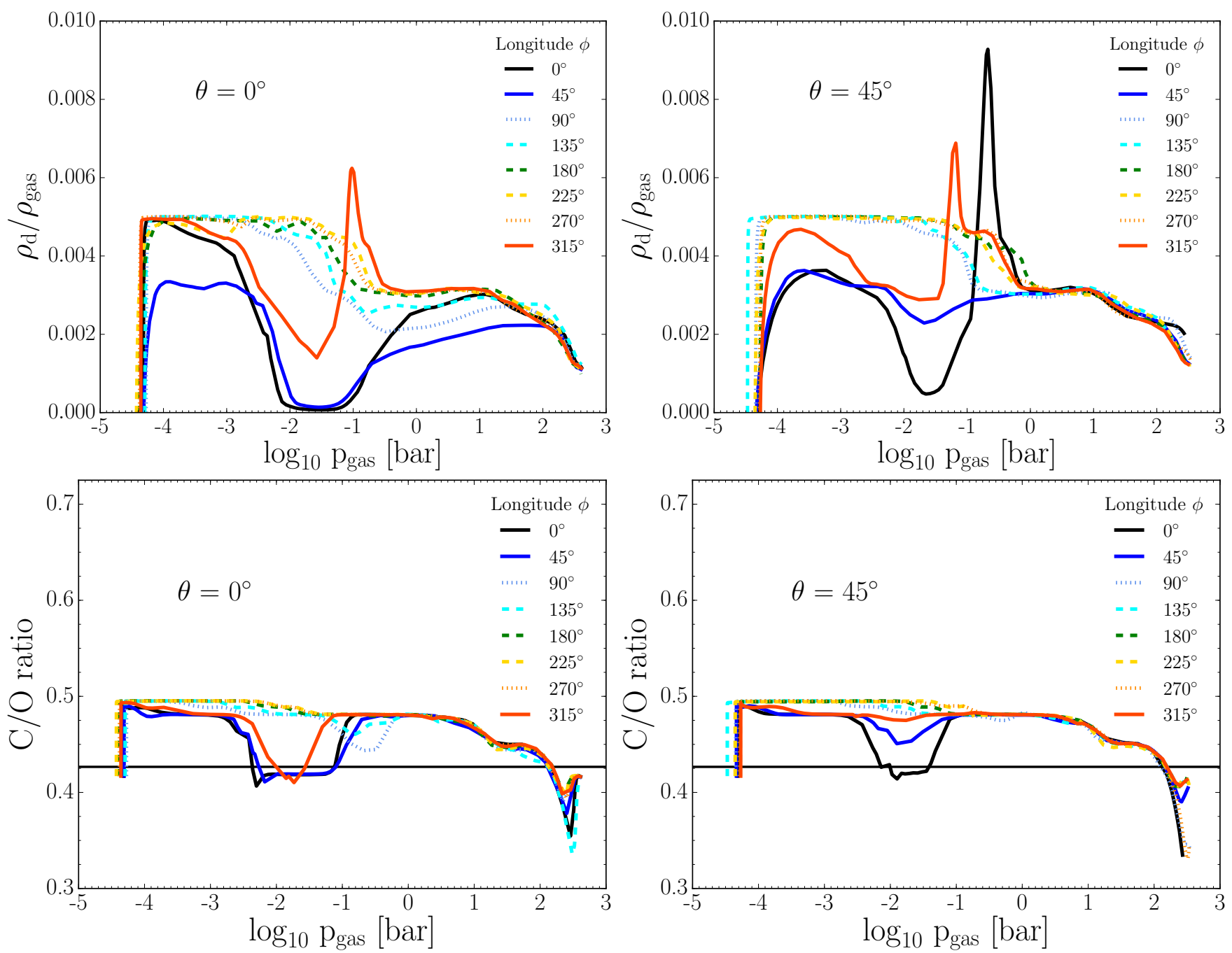

Fig. 9. Gas properties as a function of gas pressure for $\Delta \phi=+45^{\circ}$ longitude intervals from the cloud formation process. Top row: dust-to-gas ratio $\rho_{\mathrm{d}} / \rho_{\text {gas }}$ at latitudes $\theta=0^{\circ}$ and $45^{\circ}$. Bottom row: $\mathrm{C} / \mathrm{O}$ ratio at latitudes $\theta=0^{\circ}$ and $45^{\circ}$. Solid, dotted and dashed lines indicate dayside, day-night terminator and nightside profiles respectively. The horizontal black line indicates solar $\mathrm{C} / \mathrm{O}$ ratio. Regions of decreasing $\rho_{\mathrm{d}} / \rho_{\text {gas }}$ and $\mathrm{C} / \mathrm{O}$ indicate cloud particle evaporation.

and Ti return to solar abundance or slightly sub-solar abundance at the cloud base, where all materials have evaporated. The cloud base is enriched in $\mathrm{O}, \mathrm{Ca}, \mathrm{Mg}$ and $\mathrm{Si}$ which are $\sim 50 \%$ above solar abundance values. For nightside and day-night terminator profiles $\phi=90^{\circ}, 135^{\circ}, 180^{\circ}, 225^{\circ}, 270^{\circ} \theta=0^{\circ}, 45^{\circ}$, from $10^{-4.5} \ldots 10^{-2}$ bar, $\mathrm{Mg}$, Ti, $\mathrm{Si}, \mathrm{Al}$ and $\mathrm{Fe}$ are depleted by 4 to 8 orders of magnitude while $\mathrm{O}$ and $\mathrm{S}$ are depleted by $\sim 1$ order of magnitude and $\mathrm{Ca}$ by $\sim 10 \%$. The $\phi=90^{\circ}$ and $135^{\circ}, \theta=0^{\circ}$, $45^{\circ}$ show a return to near initial abundance at $\sim 10^{-0.5}$ bar from material evaporation. Other nightside/terminator profiles gradually return to initial abundance from $\sim 1$ bar to their respective cloud bases. Again, $\mathrm{O}, \mathrm{Ca}, \mathrm{Mg}$ and $\mathrm{Si}$ are slightly above solar abundance at the cloud base.

We calculate the dust-to-gas ratio and the $\mathrm{C} / \mathrm{O}$ ratio of our cloud structures. Figure 9 shows the local dust-to-gas ratio of the sample trajectories at latitudes $\theta=0^{\circ}$ and $45^{\circ}$ respectively. Dayside profiles show increases and decreases in dust-to-gas ratio corresponding to regions of nucleation/growth and evaporation. Nightside profiles show less cloud particle evaporation throughout the upper atmosphere, with only small changes in the dust-to-gas ratio which starts to drop off from $\sim 10^{-2}$ bar. Figure 9 shows the local gaseous $\mathrm{C} / \mathrm{O}$ ratio of our sample trajectories at latitudes $\theta=0^{\circ}$ and $45^{\circ}$ respectively. These follow similar trends to the dust-to-gas ratio. The $\mathrm{C} / \mathrm{O}$ ratio lowers where evaporation of cloud particles releases their oxygen baring materials, replenishing the local gas phase, The abundance of $\mathrm{C}$ is kept constant at $\epsilon_{\mathrm{C}}^{0}=10^{-3.45}$ (solar abundance) and is not affected by the formation of cloud particles in our model. Dayside equatorial profiles show $\mathrm{C} / \mathrm{O}$ ratio dips by $\sim 5 \%$ below solar values at pressures of $10^{-2.5} \ldots 10^{-1}$ bar. The $\phi=0^{\circ}, \theta=45^{\circ}$ profile also shows a dip below solar values at similar pressure levels. Apart from these localised regions of oxygen replenishment, the $\mathrm{C} / \mathrm{O}$ ratio remains above solar values for the majority of the atmospheric profiles; except from the cloud base, which is enriched with oxygen by $10 \%-20 \%$ for all profiles.

\subsection{Cloud property maps of HD 189733b}

Global cloud property maps of the atmosphere of HD 189733b enable the comparison between different atmospheric regions as a whole. This has implications for interpreting cloudy observations which sample different atmospheric regions. To produce a global cloud map of the atmosphere of HD 189733b we bi-cubically interpolate our cloud structure results across 

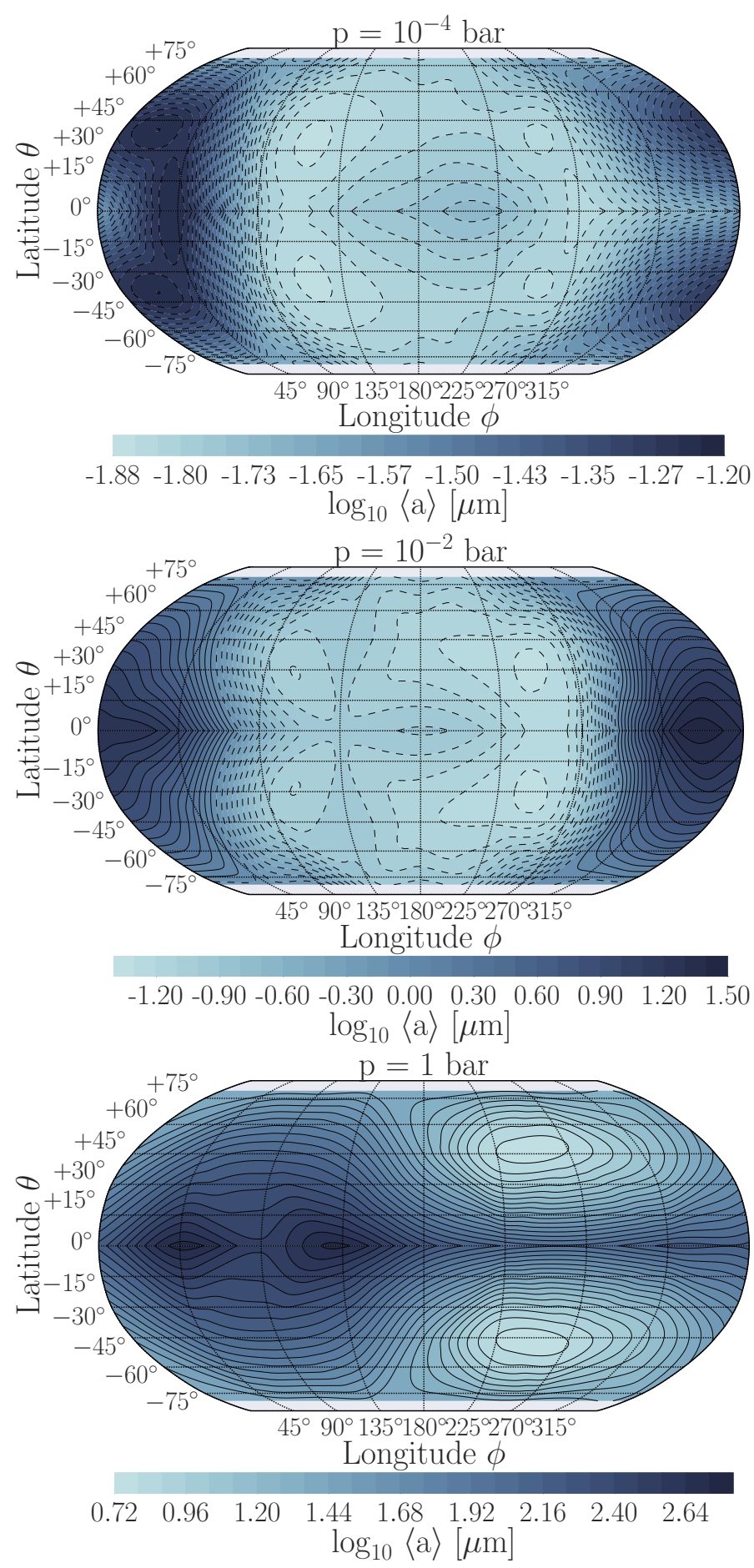
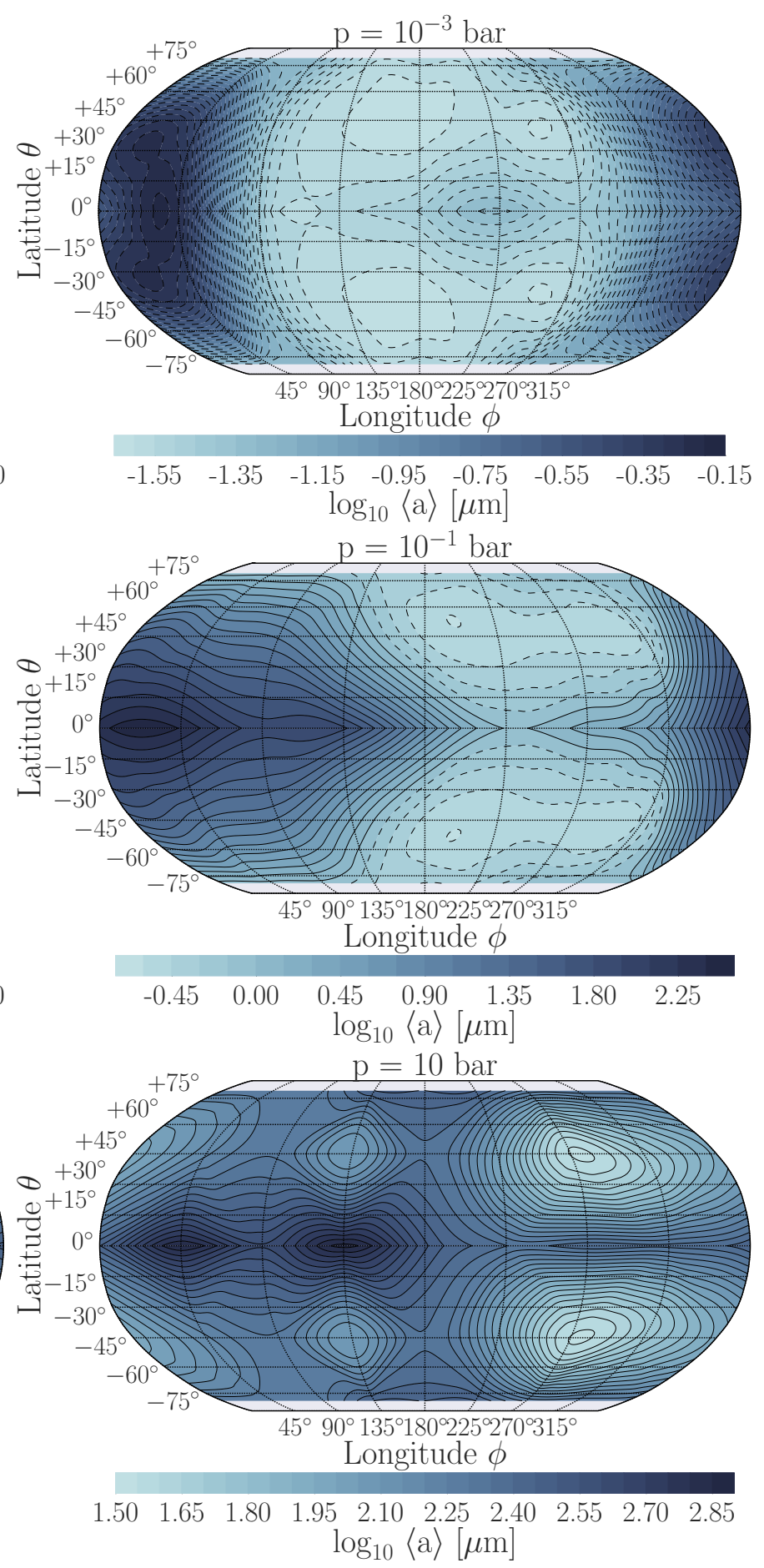

Fig. 10. Mean grain radius $\langle a\rangle[\mu \mathrm{m}]$ interpolated across the globe (assuming latitudinal (north-south) symmetry) of HD $189733 \mathrm{~b}$. Top row: $10^{-4}$ bar, $10^{-3}$ bar. Middle row: $10^{-2}$ bar, $10^{-1}$ bar. Bottom row: $1 \mathrm{bar}, 10 \mathrm{bar}$, respectively. The sub-stellar point is at $\phi=0^{\circ}, \theta=0^{\circ}$. The largest mean grain radii of cloud particles are generally found on the dayside at all pressure levels. Consequently, cloud particles on the dayside are larger than their pressure level counterparts on the nightside. Note: each plot contains a different colour scale.

longitudes $\phi=0^{\circ} \ldots 360^{\circ}$ and latitudes $\theta=0^{\circ} \ldots 80^{\circ}$. We include sample trajectories at latitude $\theta=80^{\circ}$ to interpolate to higher latitudes. We assume latitudinal (north-south) symmetry as thermodynamic conditions do not vary significantly from positive to negative latitudes (Dobbs-Dixon \& Agol 2013). Figures 10 and 11 show maps of the mean grain radius $\langle a\rangle[\mu \mathrm{m}]$ and grain number density $n_{\mathrm{d}}\left[\mathrm{cm}^{-3}\right]$ at $10^{-4}$ bar, $10^{-3}$, $10^{-2}, 10^{-1}$ bar, 1 bar and 10 bar. The mean grain radius and number density (along with material composition) are key values in calculating the wavelength dependent opacity of the clouds. At all pressures there is a contrast between the dayside and nightside mean grain radii. In the upper atmosphere $\left(<10^{-1}\right.$ bar $)$ the globally largest cloud particles occur on the dayside face. In the deeper atmosphere $\left(>10^{-1}\right.$ bar) global maximum of the cloud particle size may also occur in nightside regions. The difference between the largest and smallest grain radii at each pressure can range from 1 to 2 orders of magnitude. The highest difference in cloud particle sizes occurs at $10^{-2}$ bar where grains on the 
E. Lee et al.: Modelling the local and global cloud formation on HD $189733 \mathrm{~b}$
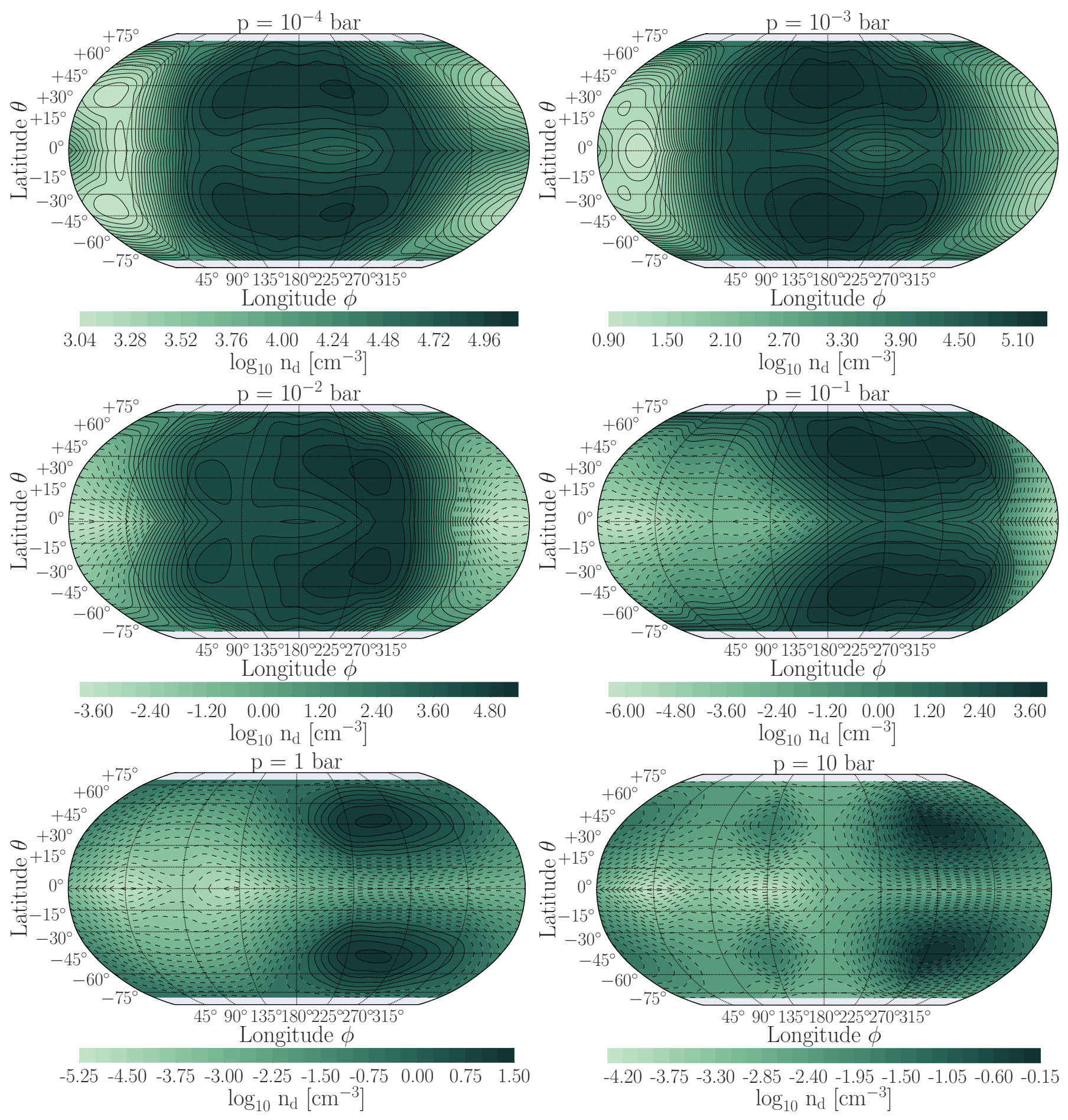

Fig. 11. Number density of cloud particles $n_{\mathrm{d}}\left[\mathrm{cm}^{-3}\right]$ interpolated across the globe (assuming latitudinal (north-south) symmetry) of HD 189733b. Top row: $10^{-4}$ bar, $10^{-3}$ bar. Middle row: $10^{-2}$ bar, $10^{-1}$ bar. Bottom row: 1 bar, 10 bar, respectively. The sub-stellar point is at $\phi=0^{\circ}, \theta=0^{\circ}$. The maximum number of cloud particles occurs on the nightside of the planet. Note: each plot contains a different colour scale.

dayside are 2 orders of magnitude bigger than the nightside. This is due to dayside profiles undergoing very efficient cloud particle surface growth at $\sim 10^{-2}$ bar, while nightside cloud particles do not grow as efficiently (Fig. 6). There are also steep gradients between the maximum and minimum mean grain radius (indicating very rapid cloud formation processes) which occur at the terminator regions $\left(\phi=90^{\circ}, 270^{\circ}\right)$ at pressure profiles $\lesssim 10^{-1}$ bar. The number density maps show similar differences between dayside and nightside profiles but with maximum values of number density generally occurring on the nightside of the planet at each pressure level. Steep gradients in number density are also present at the terminator regions. These results, taken as a whole, suggest that the wavelength dependent dust opacity significantly varies between the dayside and nightside. The mixed local composition of the cloud particles will also have an effect on the dust opacity.

\section{Cloud opacities}

We calculated the scattering and absorption properties of our cloud particles and produce global cloud opacity maps of the 
A\&A 580, A12 (2015)
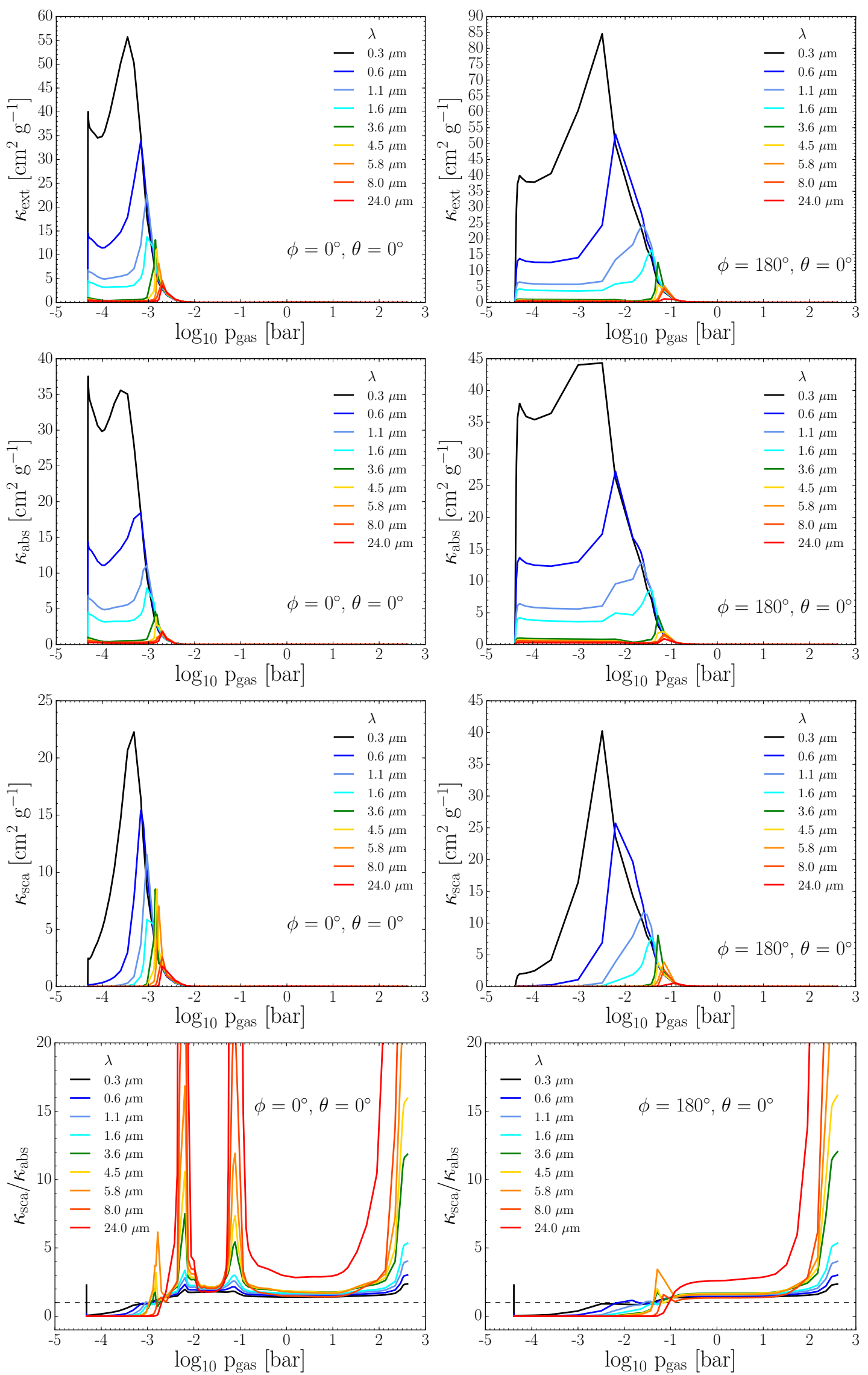

Fig. 12. Dust opacities $\kappa\left[\mathrm{cm}^{2} \mathrm{~g}^{-1}\right]$ for the cloud structure at $\phi=0^{\circ}, 180^{\circ}, \theta=0^{\circ}$ as a function of pressure. Top row: total extinction $\kappa_{\text {ext }}$. Second row: absorption $\kappa_{\text {abs }}$. Third row: scattering $\kappa_{\text {sca }}$. Bottom row: scattering to absorption ratio $\kappa_{\text {sca }} / \kappa_{\text {abs }}$. Bluer wavelengths are absorbed/scattered more efficiently than redder wavelengths in the upper atmosphere. The absorption dominates the total extinction in the upper regions and scattering in the deeper atmosphere. The spikes in scattering ratio at $10^{-2}$ and $10^{-1}$ bar correspond to $\mathrm{Fe}$ rich grain composition. 
E. Lee et al.: Modelling the local and global cloud formation on HD 189733b
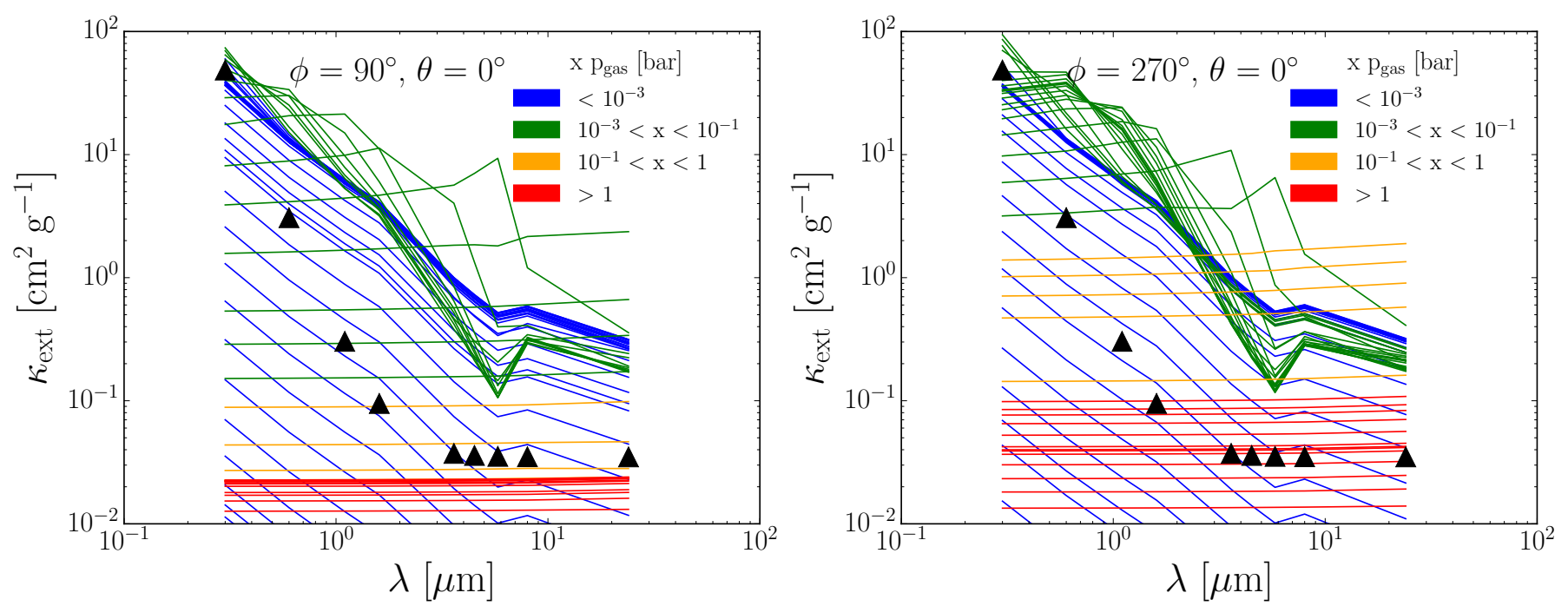

Fig. 13. Dust extinction $\kappa_{\text {ext }}\left[\mathrm{cm}^{2} \mathrm{~g}^{-1}\right]$ pressure isobars at $\phi=90^{\circ}$ (left), 270 (right), $\theta=0^{\circ}$ (equatorial dayside-nightside terminator regions). Triangles denote the values from Dobbs-Dixon \& Agol (2013) assumed additional Rayleigh slope opacity $\kappa_{\mathrm{ext}} \propto \lambda^{-4}$. Isobars follow an absorption profile $\kappa_{\text {ext }} \propto \lambda^{-2}$ at the upper atmosphere $\lesssim 10^{-1}$ bar. Isobars flatten deeper than $\gtrsim 10^{-1}$ bar.

HD 189733 b atmosphere. For an illustration of the results, we present cloud opacity calculations at longitude $\phi=0^{\circ}$ and $180^{\circ}$ at the equatorial region $\theta=0^{\circ}$. Figure 12 demonstrates that the efficiency of light extinction depends on wavelength. Bluer light is more heavily scattered/absorbed in the upper atmosphere compared to the infrared. For the $\phi=0^{\circ}$ trajectory the region of highest extinction extends from $\sim 10^{-4} \ldots 10^{-2}$ bar. The $\phi=180^{\circ}$ trajectory extends from $\sim 10^{-4} \ldots 1$ bar. The fraction of scattering to absorption is shown in Fig. 12. Absorption of the stellar light is the most efficient extinction mechanism in the upper atmosphere $\left(\sim 10^{-3}\right.$ bar $)$ while scattering dominates the extinction in the deeper atmosphere $\gtrsim 10^{-2.5}$ bar. There is a large increase in the scattering component corresponding to the $\mathrm{Fe}[\mathrm{s}]$ rich $(\sim 50 \%)$ grain regions at $10^{-2}$ and $10^{-1}$ bar in the $\phi=0^{\circ}$, $\theta=0^{\circ}$ trajectory. Between these $\mathrm{Fe}[\mathrm{s}]$ regions a $\sim 80 \% \mathrm{Al}_{2} \mathrm{O}_{3}[\mathrm{~s}]$ grain composition region occurs which is more transparent in our wavelength range than the $\mathrm{Fe}[\mathrm{s}]$ surrounding regions. For a discussion of these results, we refer to Sect. 5. From the upper atmosphere down to $\sim 10^{-3}$ bar the extinction profile follows an absorption profile of $\kappa_{\text {ext }} \propto \lambda^{-2}$. A transition region from $\sim 10^{-2}$ to $10^{-1}$ bar occurs where profiles gradually flatten from optical to infrared wavelengths. This is due to the wavelength dependent extinction efficiency of cloud particles with blue light more absorbed/scattered by small, upper atmosphere grains and red light by larger, deeper atmosphere grains. In addition, the grain composition fraction of highly opaque solid species such as $\mathrm{Fe}$ [s] increases from $\sim 10 \%$ to $\sim 20 \%$ deeper in the atmosphere $\left(>10^{-1.5}\right.$ bar).

A wavelength dependent "cloud opacity" global map can be produced by interpolation assuming latitudinal (north-south) symmetry of the cloud properties sample trajectories. We choose the $0.6 \mu \mathrm{m}$ and $5.8 \mu \mathrm{m}$ wavelengths as representative of optical and infrared wavelength extinction respectively. Figures 14 and 15 show the bi-cubic interpolated cloud particle extinction efficiency at $0.6 \mu \mathrm{m}$ and $5.8 \mu \mathrm{m}$ wavelengths across the globe from $10^{-4} \ldots 10$ bar. The optical $0.6 \mu \mathrm{m}$ cloud map shows the maximum extinction regions migrate from dayside to nightside regions with increasing pressure. The $5.8 \mu \mathrm{m}$ infrared cloud map shows the maximum extinction region also undergoes a shift, from nightside at $10^{-4}$ bar, to dayside at $10^{-3} \ldots 10^{-2}$ bar, to nightside for $<10^{-1}$ bar. The most efficient extinction region deeper in the atmosphere $\left(10^{-1} \ldots 10\right.$ bar) for both maps occurs at longitudes $\phi \sim 225^{\circ} \ldots 315^{\circ}$ which correspond to the coldest parts of the atmosphere (Fig. 2).

\subsection{Reflection and sparkling of cloud particles}

The visible appearance of our cloud particles can be estimated from their scattering properties. By estimating the relative fraction of scattered light in red, blue and green colour wavelengths, a rough RGB scale can be constructed to visualise a sparkling/reflection colour. We use the $\kappa_{\text {sca }}$ results from Sect. 4 for cloud layers at upper regions in the atmosphere $\sim 10^{-4.5} \ldots 10^{-2}$ bar at the sub-stellar point $\phi=0^{\circ}, \theta=0^{\circ}$. This profile was used as being best comparable to observations from secondary transit (occultation) observations in which the albedo (or colour) of the atmosphere can be determined (Evans et al. 2013). We linearly interpolated the $\kappa_{\text {sca }}$ to proxy RGB wavelengths and calculated their relative red, blue and green scattering fractions. This results in a deep midnight blue colour. Deeper in the atmosphere, $>10^{-3}$ bar, cloud particles scatter red, blue and green light in more equal fractions which results in redder and grayer cloud particle appearance. Helling \& Rietmeijer (2009) suggest that amorphous cloud particles can re-arrange themselves into crystalline lattice structures as they gravitationally settle. This would allow light to refract and reflect inside the cloud particle volume producing a sparkle. The sparkling colour is likely to be a similar colour to the reflected light.

\subsection{Reflectance of cloud particles}

Cloud particles have a large effect on the observable properties by reflecting incident light on the atmosphere back into space. We estimate the reflectance of the cloud particles by calculating the pressure dependent single scattering albedo $\omega_{0}$ (Bohren \& Clothiaux 2006) of the cloud particles defined as

$\omega_{0}=\frac{\kappa_{\mathrm{sca}}}{\kappa_{\mathrm{abs}}+\kappa_{\mathrm{sca}}}$ 

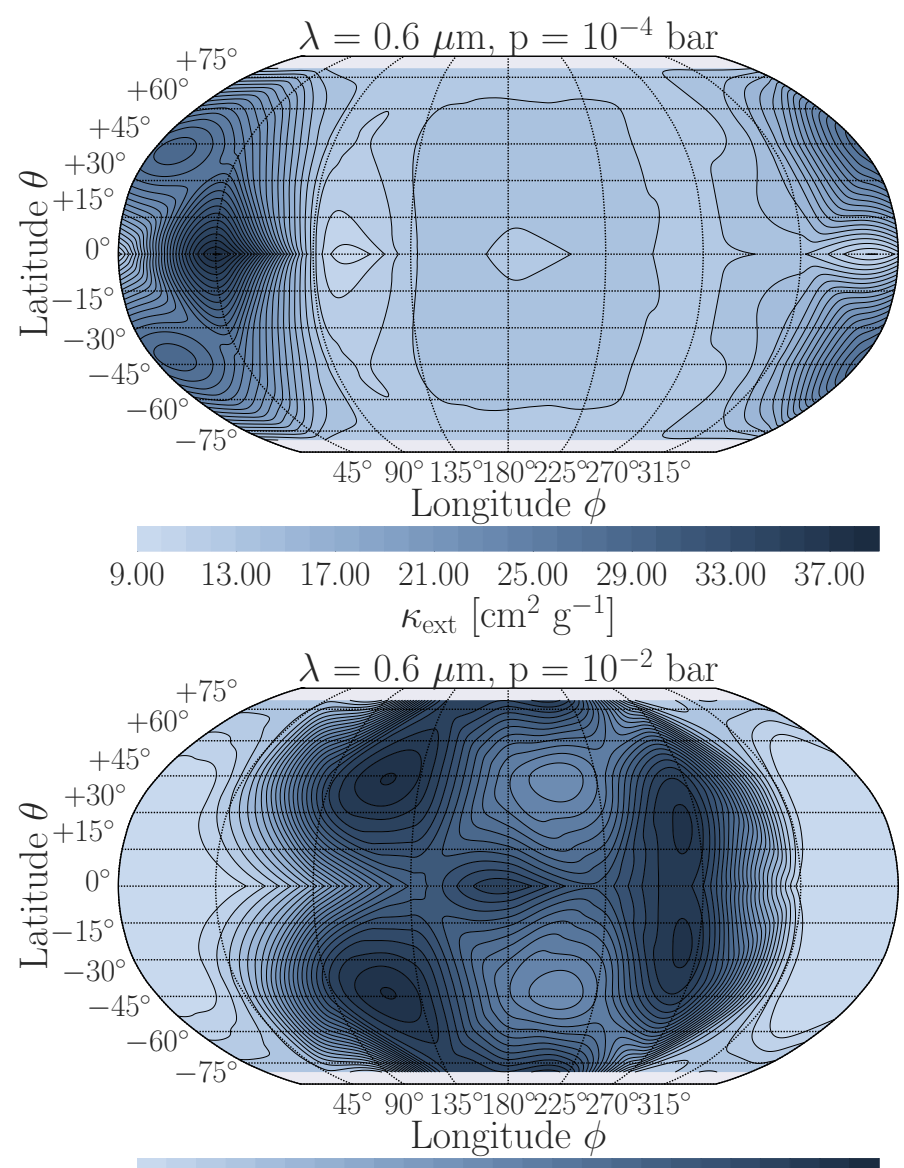

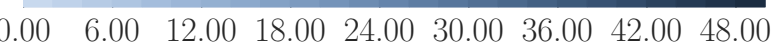
$\kappa_{\text {ext }}\left[\mathrm{cm}^{2} \mathrm{~g}^{-1}\right]$

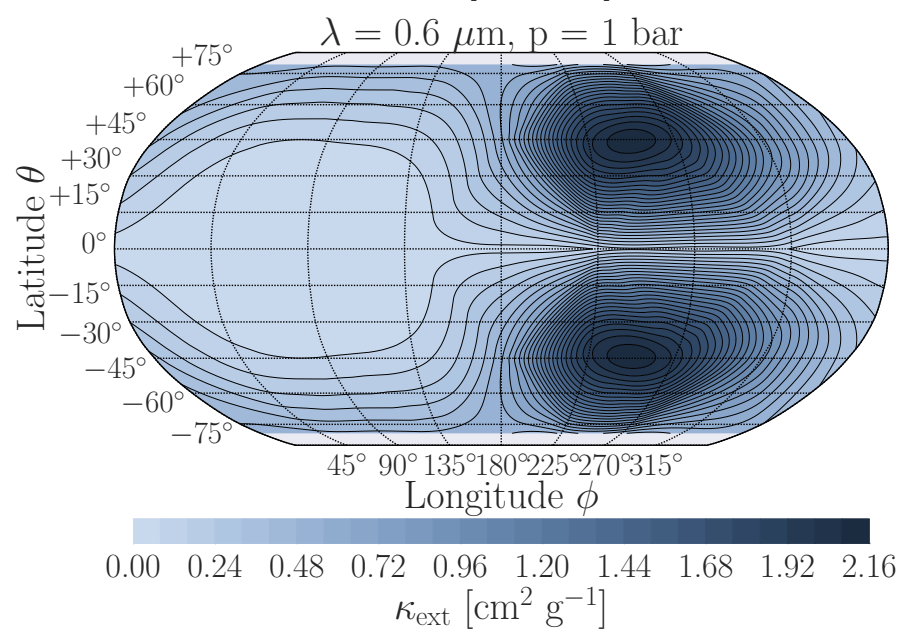

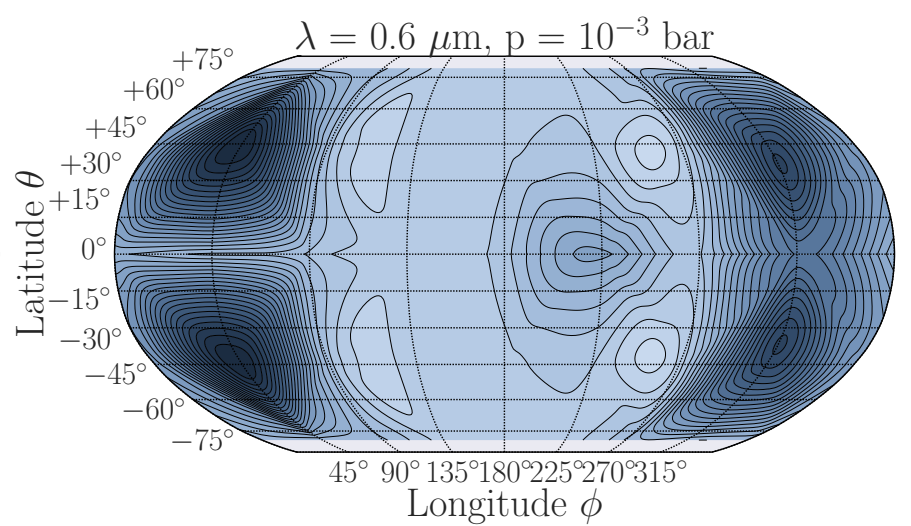

$9.00 \quad 13.50 \quad 18.00 \quad 22.50 \quad 27.00 \quad 31.50 \quad 36.00 \quad 40.50 \quad 45.00$ $\kappa_{\text {ext }}\left[\mathrm{cm}^{2} \mathrm{~g}^{-1}\right]$
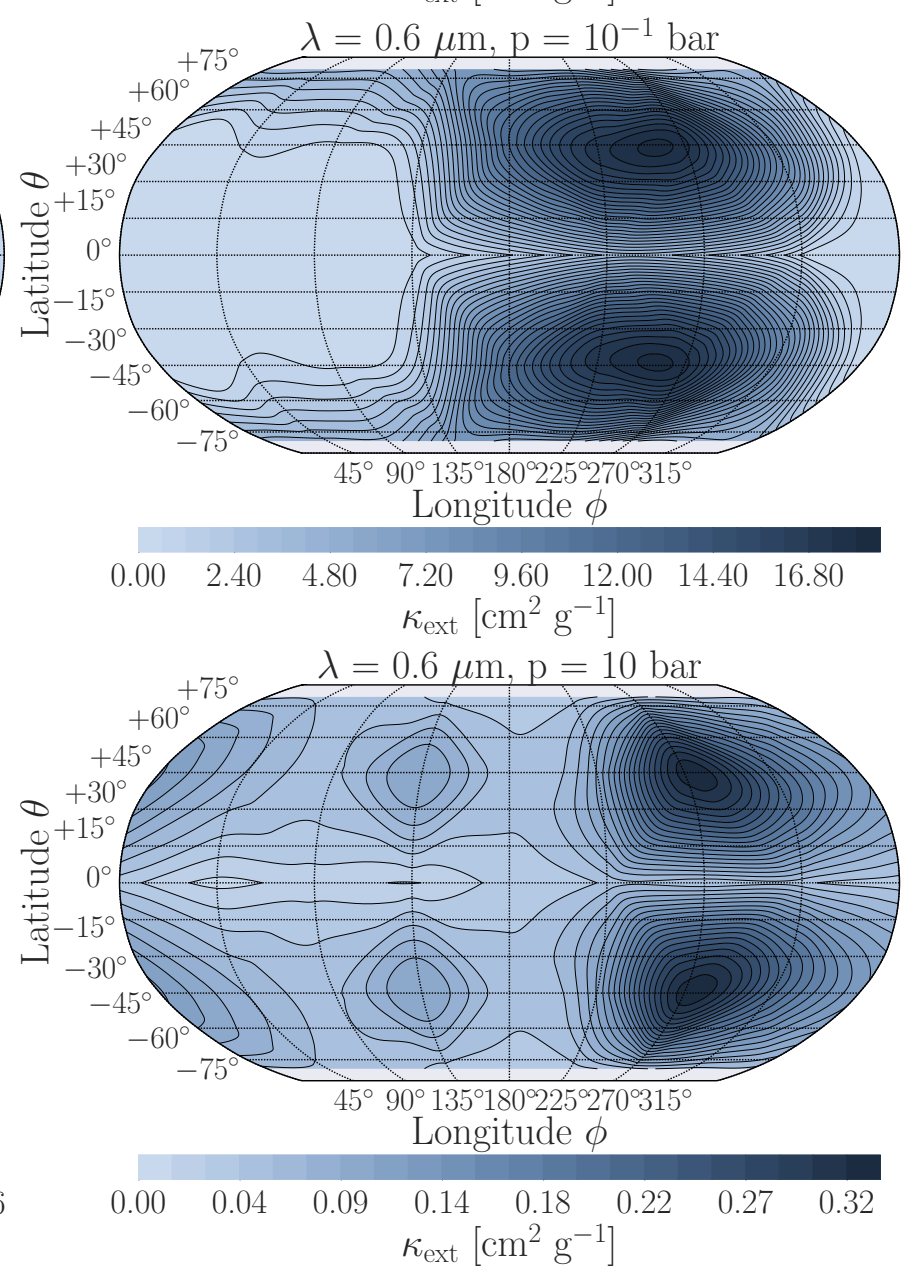

Fig. 14. Dust $\kappa_{\mathrm{ext}}\left[\mathrm{cm}^{2} \mathrm{~g}^{-1}\right]$ opacity for $0.6 \mu \mathrm{m}$ interpolated across the globe of HD 189733b assuming latitudinal (north-south) symmetry. Top row: $10^{-4}$ bar, $10^{-3}$ bar. Middle row: $10^{-2}$ bar, $10^{-1}$ bar. Bottom row: 1 bar, 10 bar, respectively. We take $0.6 \mu$ m as representative of the optical wavelength extinction due to cloud. The sub-stellar point is at $\phi=0^{\circ}, \theta=0^{\circ}$. The maximum extinction efficiency shifts from the dayside of the planet to the nightside with increasing depth. Deep in the atmosphere the most opaque region remains at $\sim 225^{\circ} \ldots 315^{\circ}$ longitudes. Note: the colour scale for each plot is different.

This ratio indicates where the cloud particles extinction is dominated by scattering $(\sim 1)$ or absorption $(\sim 0)$. Figure 16 shows maps of the calculated single scattering albedo for $8 \mu \mathrm{m}$ at $10^{-1}$ bar and 1 bar. The maximum of the reflectance occurs in the approximate longitude range $0^{\circ} \ldots 135^{\circ}$ at the equatorial region. From these maps we expect the peak of the $8 \mu \mathrm{m}$ albedo to occur from $20^{\circ} \ldots 40^{\circ}$ east of the sub-stellar point.

\section{Discussion}

Our results strongly support the idea that the Hubble and Spitzer observations of HD 189733 b described in Lecavelier Des Etangs et al. (2008), Sing et al. (2011), Gibson et al. (2012) and Pont et al. (2013) can be plausibly explained by the presence of cloud particles in the atmosphere. The cloud composition is dominated 
E. Lee et al.: Modelling the local and global cloud formation on HD 189733b
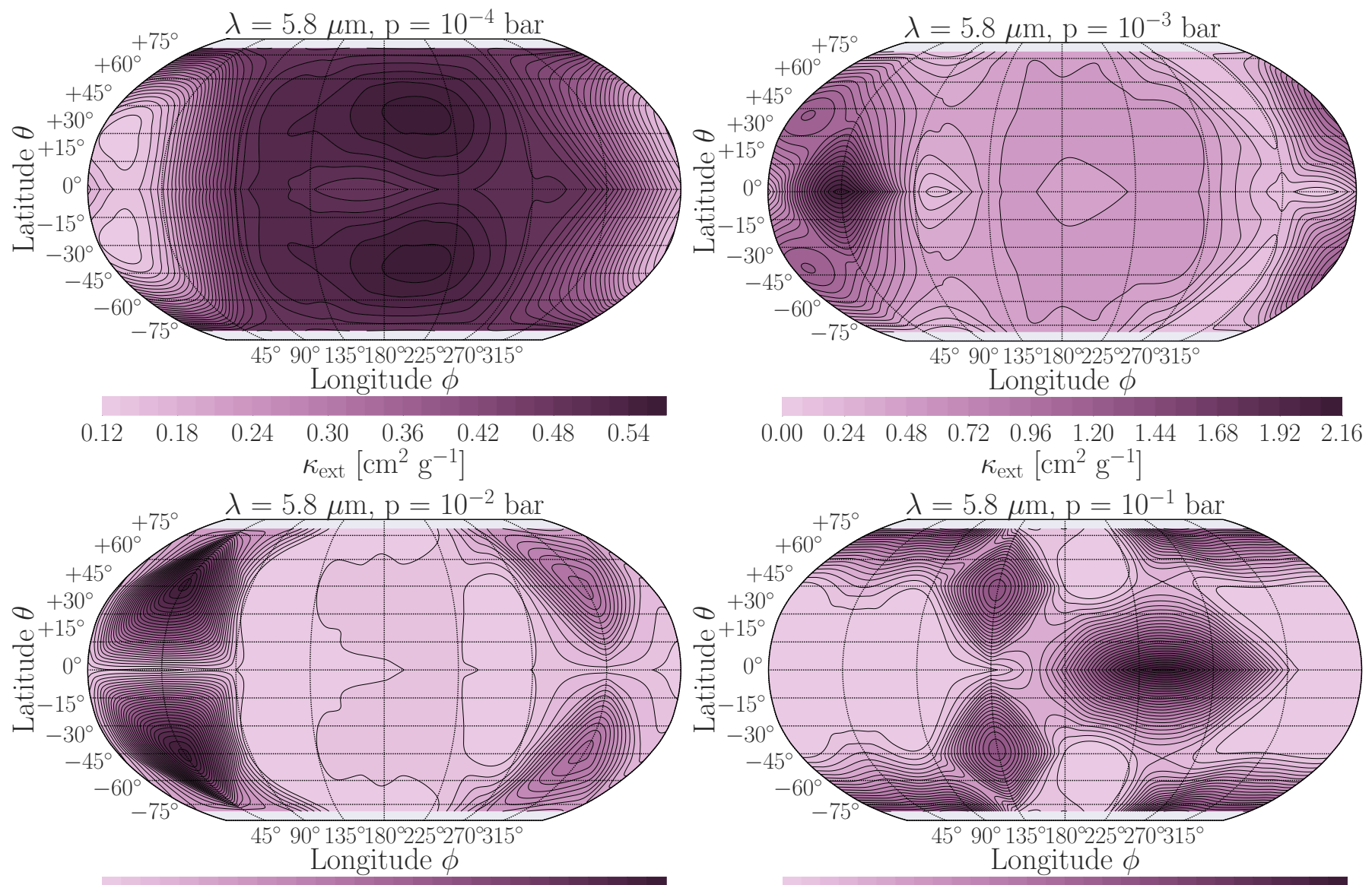

$\begin{array}{llllllllll}0.00 & 0.90 & 1.80 & 2.70 & 3.60 & 4.50 & 5.40 & 6.30 & 7.20 & 8.10\end{array}$ $\kappa_{\text {ext }}\left[\mathrm{cm}^{2} \mathrm{~g}^{-1}\right]$
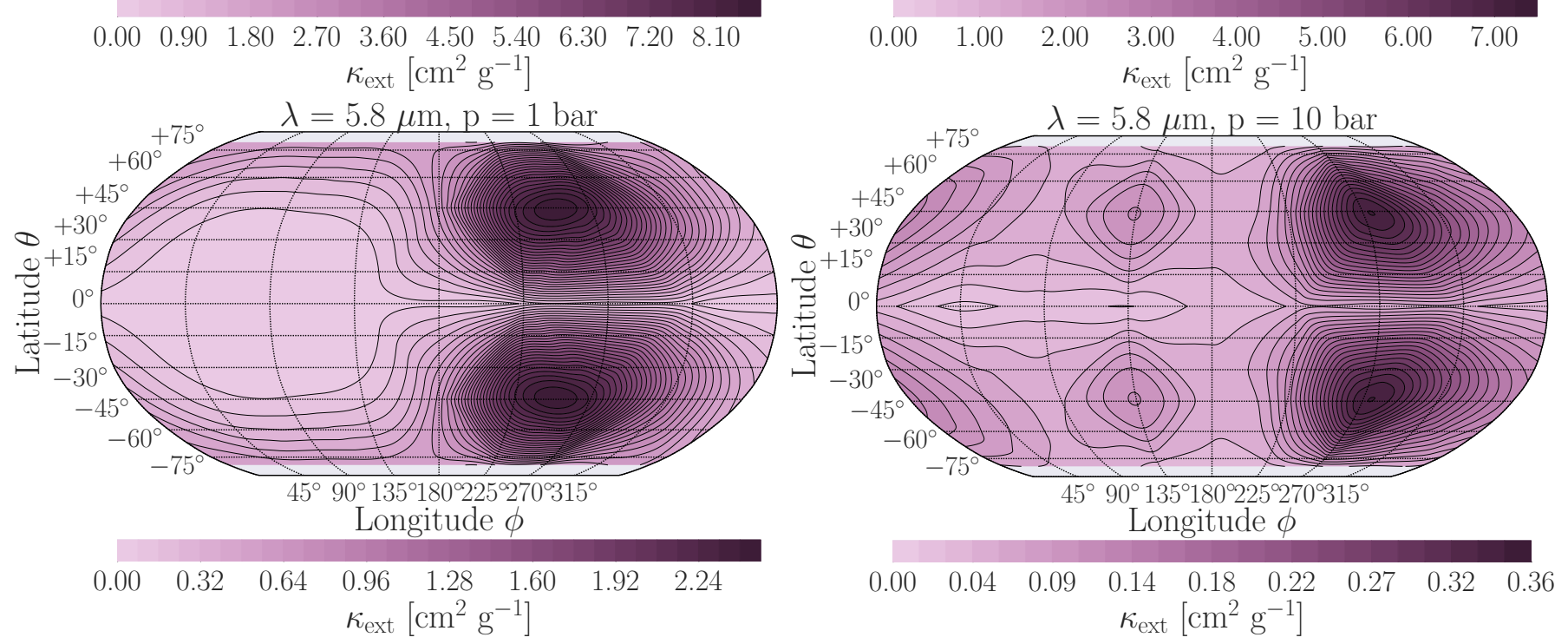

Fig. 15. Dust $\kappa_{\text {ext }}\left[\mathrm{cm}^{2} \mathrm{~g}^{-1}\right]$ opacity for $5.8 \mu \mathrm{m}$ interpolated across the globe of HD 189733b assuming latitudinal (north-south) symmetry. Top row: $10^{-4}$ bar, $10^{-3}$ bar. Middle row: $10^{-2}$ bar, $10^{-1}$ bar. Bottom row: 1 bar, 10 bar, respectively. We take $5.8 \mu$ m as representative of the infrared extinction due to cloud. The sub-stellar point is at $\phi=0^{\circ}, \theta=0^{\circ}$. The maximum extinction efficiency shifts from the dayside of the planet to the nightside with increasing depth. Deep in the atmosphere the most opaque region remains at $\sim 225^{\circ} \ldots 315^{\circ}$ longitudes. Note: the colour scale for each plot is different.

by a silicate-oxide-iron mix (Fig. 7) which supports the suggestion of a $\mathrm{MgSiO}_{3}[\mathrm{~s}]$ dominated cloudy atmosphere by Lecavelier Des Etangs et al. (2008). They suggest a $\mathrm{MgSiO}_{3}[\mathrm{~s}]$ dominated grain composition with sizes $\sim 10^{-2} \ldots 10^{-1} \mu \mathrm{m}$ at pressures of $10^{-6} \ldots 10^{-3}$ bar. Their grain size estimate is based on finding that Rayleigh scattering fits their observed feature-free slop in the optical spectral range. This analysis is consistent with our detailed model results which produce grains of $\sim 20 \% \mathrm{MgSiO}_{3}[\mathrm{~s}] \mathrm{com}-$ position with sizes $\sim 10^{-2} \ldots 10^{-1} \mu \mathrm{m}$ at $\sim 10^{-4.5} \ldots 10^{-3}$ bar, for all atmospheric profiles in this region. However, Fig. 13 shows that the isobars of the extinction $\kappa_{\text {ext }}$ at the day-night terminator regions follow an absorption profile slope in the upper atmosphere which transitions into a flat profile deeper in the atmosphere. The absorption dominated profile from $10^{-4} \ldots 10^{-3}$ bar 

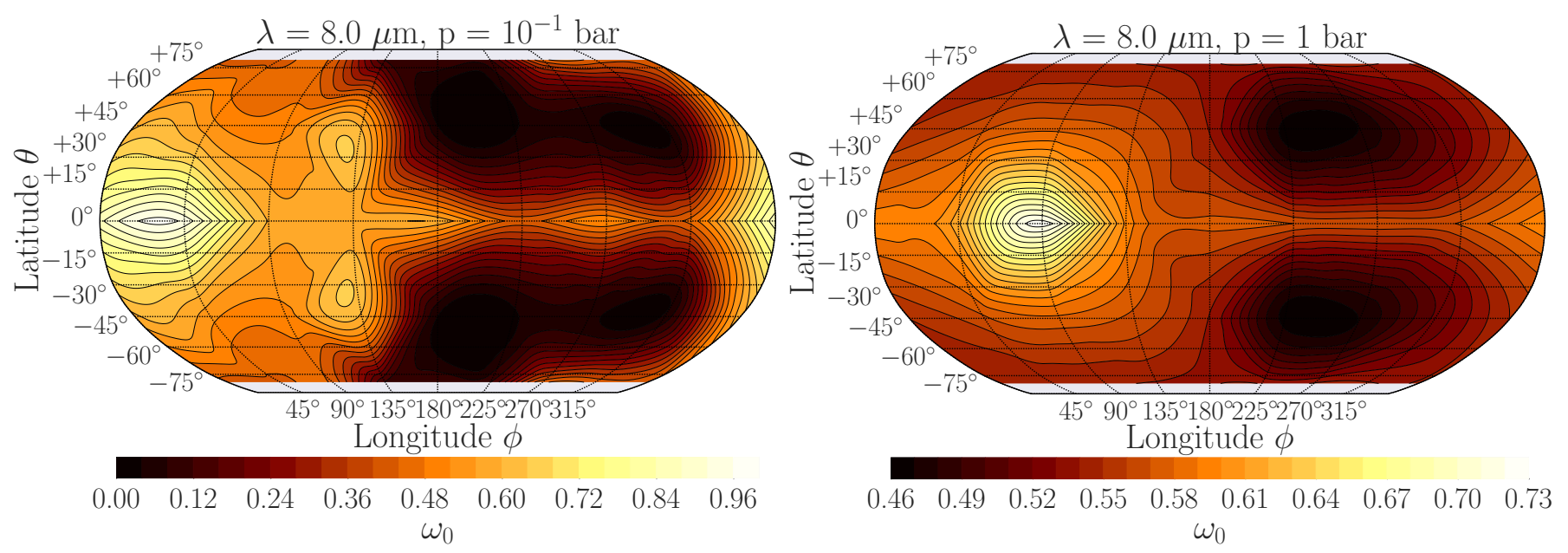

Fig. 16. Interpolated single scattering albedo $\omega_{0}$ (Eq. (19)) at a wavelength of $8 \mu \mathrm{m}$ at $10^{-1}$ bar (left), 1 bar (right) for our cloud properties. The maximum of the reflectance occurs approximately from $\phi=20^{\circ} \ldots 135^{\circ}$ longitude. These maximum reflectance regions are consistent with the $8 \mu \mathrm{m}$ Spitzer flux maps of HD $189733 \mathrm{~b}$ from Knutson et al. (2007). Note: the sub-stellar point in the diagrams is located at $\phi=0^{\circ}, \theta=0^{\circ}$, at the left side of the figures.

does not support the Rayleigh scattering parameterisations for the observations of HD 189733b. This does not rule out cloud particles as the source of the Rayleigh slope. A possibility is that many smaller grains form at lower pressure regions than the boundary applied. This would increase the scattering component of the cloud opacity. Furthermore, in a 3D atmosphere, small grains may be more easily lofted than larger grains in regions of upward gas flow. Our static 1D model does not include the 3D effects of a dynamic, changing atmosphere.

Section 3.4 shows the effect that cloud particles have on local element abundances. Depletion of elements occur when they are consumed by cloud particle formation and replenishment occurs when these cloud particles evaporate. In our cloud model the elements that constitute the main cloud particle composition were depleted by 3 orders of magnitude or more from $\sim 10^{-4.5} \ldots 10^{-2}$ bar. These results suggest that the presence of cloud particles would generally flatten the spectral signatures from these elements and associated molecules in the atmosphere. The evaporation of molecules from cloud particles transports elements from upper to lower atmospheric height. This suggests that elements thought to contribute to upper atmosphere thermal inversion layers such as $\mathrm{Ti}$ (more specifically TiO; Fortney et al. 2008; Spiegel et al. 2009; Showman et al. 2009) are transported to the deeper atmosphere by cloud particles. The depletion and movement of $\mathrm{TiO}$ from the upper to lower atmosphere could reduce the intensity of a stratospheric inversion and/or move it deeper into the atmosphere or destroy it completely. Figure 9 shows that regions of the atmosphere can be oxygen poor or rich depending on the cloud processes. This significantly alters the $\mathrm{C} / \mathrm{O}$ ratio, where it is increased by the growth of oxygen baring solid materials onto cloud particle surfaces and decreased when these materials evaporate back into the gas phase. This could have an impact on interpreting observations of over/under abundant $\mathrm{C} / \mathrm{O}$ ratios in exoplanet atmospheres as well as altering the local chemistry in oxygen depleted regions.

The opacity of the clouds will strongly influence the spectral signature from the atmosphere by absorbing or scattering various wavelengths at different efficiency. From Fig. 12 optical wavelengths are preferentially absorbed and scattered in the upper atmosphere. We therefore suggest that mineral clouds are responsible for the planets observed albedo and deep blue colour suggested by Berdyugina et al. (2011) and Evans et al. (2013). The migration of the maximum efficiency of the cloud extinction from dayside to nightside (Figs. 14, 15) at $0.6 \mu \mathrm{m}$ and $5.8 \mu \mathrm{m}$ shows a strong dayside-nightside opacity contrast. The terminator regions at longitude $\phi=90^{\circ}$ and $270^{\circ}$ also show differences in dust extinction efficiency, especially deeper in the atmosphere. Different thermodynamic conditions result in mean grain sizes, compositions and opacity that is different at each terminator region. This has consequences for interpreting transmission spectroscopy measurements since observations of opposite limbs of the planet would have different extinction properties. In addition, the cloud maps also show steep gradients of cloud properties at the terminator regions. Transit spectroscopy observations that measure these regions would sample a variety of cloud particle number density, sizes and distributions dependent on wavelength. In Fig. 7 the profiles at $\phi=0^{\circ}$ and $45^{\circ}$ show a region compositionally dominated by $\mathrm{Al}_{2} \mathrm{O}_{3}$ [s] cloud particles from $\sim 10^{-2} \ldots 10^{-1}$ bar with $\mathrm{Fe}[\mathrm{s}]$-rich grains on above and below this region. The effect of this $\mathrm{Al}_{2} \mathrm{O}_{3}$ [s] region is to produce a locally lower cloud opacity layer flanked by high opacity regions (Fig. 12). This would have a significant effect on radiation propagation through the atmosphere as the $\mathrm{Fe}[\mathrm{s}]$ rich grains could shield photons reaching the $\mathrm{Al}_{2} \mathrm{O}_{3}$ [s] layer from above or below.

Our qualitative calculation of the cloud contribution to the albedo provides yet another insight into the observational consequences of our cloud modelling. Our calculated single scattering albedo for the $8 \mu \mathrm{m}$ band (Fig. 16) show that the maximum reflectance occurs approximately in the longitude range $0^{\circ} \ldots 135^{\circ}$ at the equator at $10^{-1}$ and 1 bar. With the peak occurring from $20^{\circ} \ldots 40^{\circ}$. These maximum reflectance regions reproduce the areas of maximum $8 \mu \mathrm{m}$ flux map from Knutson et al. (2007). This suggests that cloud particles influence observed phase curves of hot Jupiters depending on the position and reflectivity of cloud particles in the atmosphere. These effects have been observed for hot Jupiter Kepler 7-b (Demory et al. 2013), where Kepler observations showed a westward shift in optical phase curves (GCM/RHD models predict an eastward shift). Spitzer phase curves showed that the shift was non-thermal in origin. This shift was attributed to non-uniform, longitude dependent optical scattering from clouds. Our $8 \mu \mathrm{m}$ reflectance result, albeit qualitatively, show that clouds can contribute to the infrared flux 
E. Lee et al.: Modelling the local and global cloud formation on HD 189733b

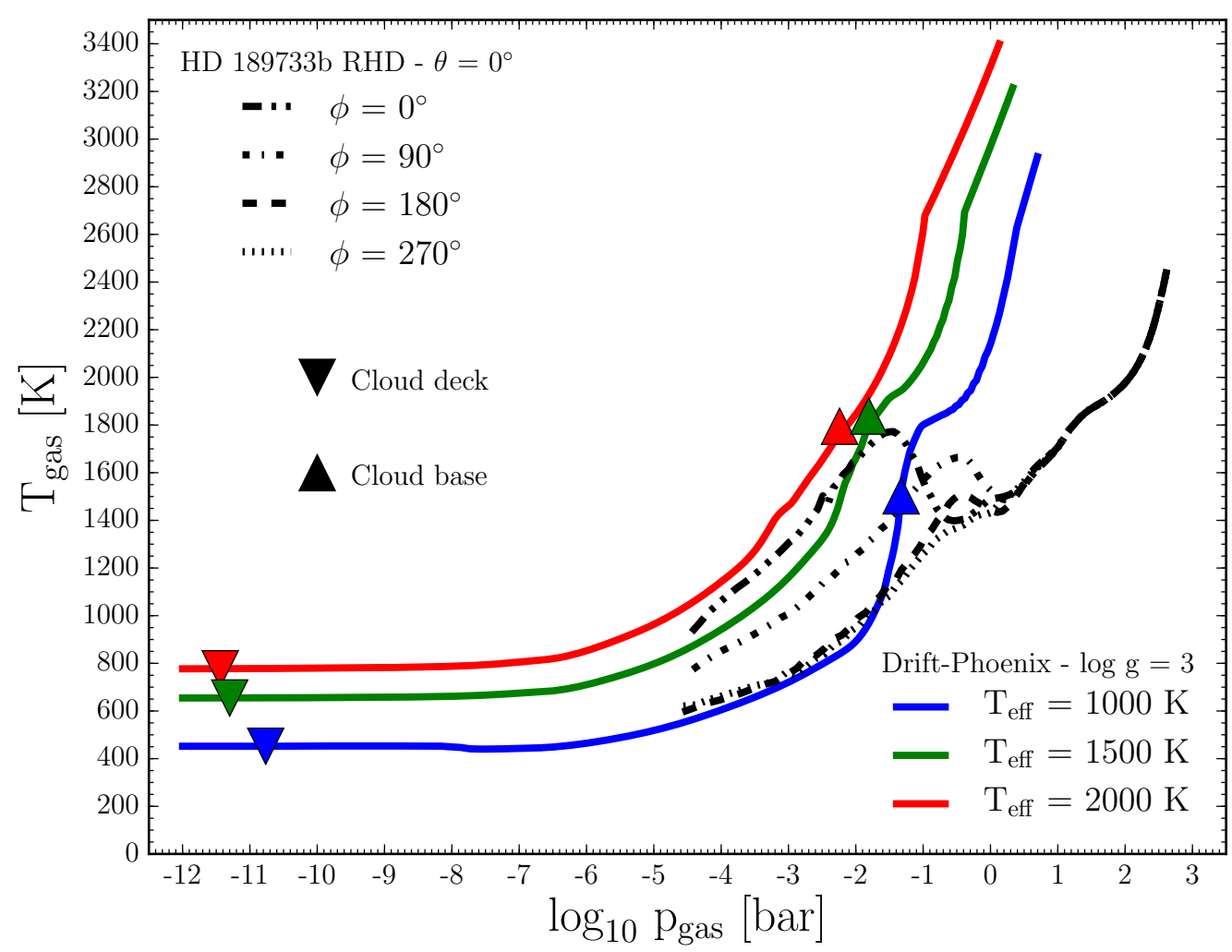

Fig. 17. $\left(T_{\text {gas }}, p_{\text {gas }}\right)$ profiles for DRIFT-PHOENIX atmosphere models (coloured, solid) for $\log g=3, T_{\text {eff }}=1000,1500,2000 \mathrm{~K}$ and the Dobbs-Dixon $\&$ Agol (2013) RHD model at $\phi=0^{\circ}, 90^{\circ}, 180^{\circ}, 270^{\circ}, \theta=0^{\circ}$ (black, styled). Downward and upward facing triangles denote the cloud deck and base respectively. The DRIFT-PHOENIX profiles show that cloud formation begins at $\sim 10^{-11}$ bar.

observed from the exoplanet atmosphere. It may not be simple to disentangle contributions by thermal emission and scattering/reflection clouds from infrared observational properties.

Figure 17 shows a $\left(T_{\text {gas }}, p_{\text {gas }}\right)$ structure comparison between DRIFT-PHOENIX $\left(\log g=3, T_{\text {eff }}=1000,1500,2000 \mathrm{~K}\right)$ and the Dobbs-Dixon \& Agol (2013) 3D RHD model. Comparing trajectories from the 3D RHD model and the 1D Drift-Phoenix models suggests that clouds could be more extended into the lowerpressure regions than our present results show. Previous studies (e.g. DRIFT-PHOENIX; Witte et al. 2011; Woitke \& Helling 2004) involving cloud formation, the lower pressure boundary can be up to $\sim 10^{-12}$ bar with cloud formation occurring from $\sim 10^{-11}$ bar, these studies contain a smoother non-cloud to cloudy upper atmospheric region than our present results. The cloud deck in the DRIFT-PHOENIX models starts from $\sim 10^{-11}$ bar, 7 orders of magnitude lower that the RHD upper boundary pressure. Therefore, our results do not capture cloud formation outside the RHD model boundary conditions yet. We also suggest that cloud formation can continue further inward than the results presented here. Due to the increasing density and high pressure, cloud particle material remains thermally stable until considerably higher temperatures and survives deeper into the atmosphere.

We probed a 3D hot Jupiter atmospheric structure, irradiated by a host star, through selecting $161 \mathrm{D}$ atmospheres trajectories along the equator and latitude $\theta=45^{\circ}$. We aimed to capture the main features of a dynamic atmosphere like eastwest jet streams, latitudinal differences and dayside-nightside differences. We used these thermodynamic and velocity profiles to study cloud formation in order to present consistently calculated cloud properties for HD 189733b. Our ansatz does not yet include a self-consistent feedback onto the $\left(T_{\text {gas }}, p_{\text {gas }}\right.$, $\boldsymbol{v}_{\text {gas }}$ ) structure due to radiative transfer processes. We note that due to an additional wavelength dependent dust opacity term in the radiative transfer scheme of Dobbs-Dixon \& Agol (2013), this feedback is already accounted for to a significant degree in the current $\left(T_{\text {gas }}, p_{\text {gas }}\right)$ profiles. Furthermore, we do not include non-LTE kinetic gas-phase chemistry such as photochemistry. Departures from LTE have been detailed in non-equilibrium models of HD 189733b's atmosphere performed by Moses et al. (2011), Venot et al. (2012), Agúndez et al. (2014) using thermodynamic input from the Showman et al. (2009) global circulation model.

\section{Conclusion and summary}

We have presented the first spatially varying kinetic cloud simulation of the irradiated hot Jupiter exoplanet HD 189733b. We applied a 2-model approach with our cloud formation model using 1D thermodynamic input from a 3D RHD simulation of HD 189733b. Our results suggest that HD 189733b has a significant cloud component in the atmosphere, spanning a large pressure scale throughout the entire globe. Cloud particles remain thermally stable deep in the atmosphere up to $\sim 10$ bar pressures and reach $\sim$ mm sizes at the cloud base. We suggest that cloud particles form at a lower pressure boundary than considered here, and also survive to deeper depths. Cloud properties change significantly from dayside to nightside and from equator to mid latitudes with variations in grain size, number density and wavelength dependent opacity. The cloud property maps show that steep gradients between dayside and nightside cloud proprieties occur at dayside-nightside transition regions. These cloud property differences have implications on interpreting observations of HD 189733b, depending what region and depth of the planet 
is probed. The single scattering albedo calculations showed that cloud particles could play a significant role in planetary phase curves. The reflectance of the clouds at $8 \mu \mathrm{m}$ showed that a significant fraction of infrared flux could originate from scattering/reflecting cloud particles. Since the most reflective clouds in the infrared correspond to regions of highest temperature it may be difficult to distinguish between cloud reflections and thermal emission. The extinction properties of the cloud particles exhibit absorption signatures in the upper atmosphere $\left(\lesssim 10^{-2}\right.$ bar $)$ which flatten deeper in the atmosphere $\left(\gtrsim 10^{-1}\right.$ bar $)$. However, this does not rule out a cloud particle origin for the observations of Rayleigh scattering. The scattering properties also suggest that the cloud particles would sparkle/reflect a midnight blue colour over the optical wavelength regime.

Acknowledgements. E.L. and Ch.H. highlight the financial support of the European community under the FP7 ERC starting grant 257431. Our respective local computer support is highly acknowledged. We thank P. Woitke, C.R. Stark and P. Rimmer for helpful discussions and feedback. We thank L. Neary for insightful discussion on atmospheric mixing.

\section{References}

Agol, E., Cowan, N. B., Knutson, H. A., et al. 2010, ApJ, 721, 1861

Agúndez, M., Parmentier, V., Venot, O., Hersant, F., \& Selsis, F. 2014, A\&A, 564, A73

Anders, E., \& Grevesse, N. 1989, Geochim. Cosmochim. Acta, 53, 197

Bailey, R. L., Helling, C., Hodosán, G., Bilger, C., \& Stark, C. R. 2014, ApJ, 784,43

Barman, T. S., Hauschildt, P. H., \& Allard, F. 2001, ApJ, 556, 885

Berdyugina, S. V., Berdyugin, A. V., Fluri, D. M., \& Piirola, V. 2011, ApJ, 728, L6

Bohren, C. F., \& Clothiaux, E. E. 2006, Fundamentals of Atmospheric Radiation, eds. C. F. Bohren, \& E. E. Clothiaux, 450

Bohren, C. F., \& Huffman, D. R. 1983, Absorption and scattering of light by small particles (New York: Wiley)

Bruggeman, D. A. G. 1935, Annalen der Physik, 416, 636

Charbonneau, D., Knutson, H. A., Barman, T., et al. 2008, ApJ, 686, 1341

Danielski, C., Deroo, P., Waldmann, I. P., et al. 2014, ApJ, 785, 35

Dehn, M., Helling, C., Woitke, P., \& Hauschildt, P. 2007, in IAU Symp., eds. F. Kupka, I. Roxburgh, \& K. L. Chan, 239, 227

Demory, B.-O., de Wit, J., Lewis, N., et al. 2013, ApJ, 776, L25

Désert, J.-M., Sing, D., Vidal-Madjar, A., et al. 2011, A\&A, 526, A12

Dobbs-Dixon, I., \& Agol, E. 2013, MNRAS, 435, 3159

Dorschner, J., Begemann, B., Henning, T., Jaeger, C., \& Mutschke, H. 1995, A\&A, 300, 503

Evans, T. M., Pont, F., Sing, D. K., et al. 2013, ApJ, 772, L16

Fortney, J. J., Lodders, K., Marley, M. S., \& Freedman, R. S. 2008, ApJ, 678, 1419

Fortney, J. J., Shabram, M., Showman, A. P., et al. 2010, ApJ, 709, 1396
Gail, H.-P., \& Sedlmayr, E. 1986, A\&A, 166, 225

Gibson, N. P., Aigrain, S., Pont, F., et al. 2012, MNRAS, 422, 753

Grillmair, C. J., Burrows, A., Charbonneau, D., et al. 2008, Nature, 456, 767

Hartogh, P., Medvedev, A. S., Kuroda, T., et al. 2005, J. Geophy. Res. (Planets), 110, 11008

Helling, C., \& Fomins, A. 2013, Roy. Soc. London Philos. Trans. Ser. A, 371, 10581

Helling, C., \& Rietmeijer, F. J. M. 2009, Inter. J. Astrobiol., 8, 3

Helling, C., \& Woitke, P. 2006, A\&A, 455, 325

Helling, C., Klein, R., Woitke, P., Nowak, U., \& Sedlmayr, E. 2004, A\&A, 423, 657

Helling, C., Dehn, M., Woitke, P., \& Hauschildt, P. H. 2008a, ApJ, 677, L157

Helling, C., Woitke, P., \& Thi, W.-F. 2008b, A\&A, 485, 547

Helling, C., Jardine, M., \& Mokler, F. 2011, ApJ, 737, 38

Henning, T., Begemann, B., Mutschke, H., \& Dorschner, J. 1995, A\&AS, 112, 143

Huitson, C. M., Sing, D. K., Vidal-Madjar, A., et al. 2012, MNRAS, 422, 2477

Jäger, C., Dorschner, J., Mutschke, H., Posch, T., \& Henning, T. 2003, A\&A, 408, 193

Jeong, K. S., Winters, J. M., Le Bertre, T., \& Sedlmayr, E. 2003, A\&A, 407, 191

Knutson, H. A., Charbonneau, D., Allen, L. E., et al. 2007, Nature, 447, 183

Knutson, H. A., Lewis, N., Fortney, J. J., et al. 2012, ApJ, 754, 22

Kreidberg, L., Bean, J. L., Désert, J.-M., et al. 2014, Nature, 505, 69

Lecavelier Des Etangs, A., Pont, F., Vidal-Madjar, A., \& Sing, D. 2008, A\&A, 481, L83

Lee, G., Helling, C., Giles, H., \& Bromley, S. T. 2015, A\&A, 575, A11

Ludwig, H.-G., Allard, F., \& Hauschildt, P. H. 2002, A\&A, 395, 99

McCullough, P. R., Crouzet, N., Deming, D., \& Madhusudhan, N. 2014, ApJ, 791,55

Mie, G. 1908, Ann. Phys., 330, 377

Moses, J. I., Visscher, C., Fortney, J. J., et al. 2011, ApJ, 737, 15

Palik, E. D. 1985, Handbook of optical constants of solids (New York: Academic Press)

Parmentier, V., Showman, A. P., \& Lian, Y. 2013, A\&A, 558, A91

Pont, F., Sing, D. K., Gibson, N. P., et al. 2013, MNRAS, 432, 2917

Posch, T., Kerschbaum, F., Fabian, D., et al. 2003, ApJS, 149, 437

Rimmer, P. B., \& Helling, C. 2013, ApJ, 774, 108

Showman, A. P., \& Polvani, L. M. 2011, ApJ, 738, 71

Showman, A. P., Fortney, J. J., Lian, Y., et al. 2009, ApJ, 699, 564

Sing, D. K., Pont, F., Aigrain, S., et al. 2011, MNRAS, 416, 1443

Spiegel, D. S., Silverio, K., \& Burrows, A. 2009, ApJ, 699, 1487

Stark, C. R., Helling, C., Diver, D. A., \& Rimmer, P. B. 2014, Int. J. Astrobiol., 13,165

Swain, M. R., Vasisht, G., \& Tinetti, G. 2008, Nature, 452, 329

Swain, M. R., Vasisht, G., Tinetti, G., et al. 2009, ApJ, 690, L114

Tinetti, G., Vidal-Madjar, A., Liang, M.-C., et al. 2007, Nature, 448, 169

Tsai, S.-M., Dobbs-Dixon, I., \& Gu, P.-G. 2014, ApJ, 793, 141

Venot, O., Hébrard, E., Agúndez, M., et al. 2012, A\&A, 546, A43

Wakeford, H. R., \& Sing, D. K. 2015, A\&A, 573, A122

Witte, S., Helling, C., \& Hauschildt, P. H. 2009, A\&A, 506, 1367

Witte, S., Helling, C., Barman, T., Heidrich, N., \& Hauschildt, P. H. 2011, A\&A, 529, A44

Woitke, P., \& Helling, C. 2003, A\&A, 399, 297

Woitke, P., \& Helling, C. 2004, A\&A, 414, 335

Zeidler, S., Posch, T., \& Mutschke, H. 2013, A\&A, 553, A81 